\title{
Diffusion-induced spatio-temporal oscillations in an epidemic model with two delays
}

\author{
Yanfei Du \\ Shaanxi University of Science and Technology, Xi'an 710021, China. \\ Ben Niu*, Junjie Wei \\ Department of Mathematics, Harbin Institute of Technology, Weihai 264209, China. \\ *Corresponding author, niu@hit.edu.cn
}

(Dated: July 7, 2021)

\begin{abstract}
We investigate a diffusive, stage-structured epidemic model with the maturation delay and freelymoving delay. Choosing delays and diffusive rates as bifurcation parameters, the only possible way to destabilize the endemic equilibrium is through Hopf bifurcation. The normal forms of Hopf bifurcations on the center manifold are calculated, and explicit formulae determining the criticality of bifurcations are derived. There are two different kinds of stable oscillations near the first bifurcation: on one hand, we theoretically prove that when the diffusion rate of infected immature individuals is sufficiently small or sufficiently large, the first branch of Hopf bifurcating solutions is always spatially homogeneous; on the other, fixing this diffusion rate at an appropriate size, stable oscillations with different spatial profiles are observed, and the conditions to guarantee the existence of such solutions are given. These phenomena are investigated by calculating the corresponding eigenfunction of the Laplacian at the first Hopf bifurcation point.
\end{abstract}

Keywords: Epidemic model; Stage structure; Delay; Diffusion; Hopf bifurcation; Spatio-temporal oscillation 


\section{INTRODUCTION}

Kermack and McKendrick [1] proposed a classical epidemic model in a closed population consisting of susceptible, infected, and recovered classes, with sizes denoted by $S(t), I(t)$ and $R(t)$ at time $t$, respectively. From then on, epidemic models have received much attention from many authors [2 8 . Taking into account the fact that some diseases have an incubation period, the exposed have been introduced into epidemic models, and SEIR epidemic models have been studied [2]. Since the recovered may lose immunity and return to be susceptible, SIRS models have been proposed to describe the phenomenon [3, 4]. Other types of epidemic models have also been studied, such as SIS, SEIRS, SEIS models and so on [5] 7]. In epidemic models, the incidence rate plays a significant role in the spread of diseases, which describes how the susceptible individuals contact with the infective individuals and become infectious. The bilinear incidence rate of form $\lambda S I$ is assumed that the infection occurs proportionally to the sizes of the susceptible and the infected. Other incidence rates include the proportionate mixing incidence rate, nonlinear incidence rate, and saturation incidence rate. For details, readers are referred to [8 10] and the references therein. An important question to be answered in studying an infectious disease is when the disease will be persistent, and when it will die out. It turns out that the basic reproduction ratio $R_{0}$ is usually a sharp threshold which determines the global dynamics of the disease. If $R_{0}<1$, the disease-free steady state is stable, while if $R_{0}>1$ there exists at least one endemic steady state, which is stable [8, 11, 12].

The effect of delay on the dynamics of disease transmission models has attracted much attention. Cooke [13] proposed a vector disease model with a discrete time delay. Grossman [14] constructed SIR epidemic models with discrete delays. After their work, different kinds of time lags have been incorporated in epidemic models. Since many infectious diseases take a period of time to appear some symptoms and become infectious after infected (namely incubation period), Saker [15] studied an epidemic model with incubation delay. Khan and Krishnan [16] took time delay into account in the recruitment of infected persons, it turned out that the introduction of a time delay into the transmission term can destabilize the system and periodic solutions can arise by Hopf bifurcation. To describe the fact that vaccines do not immediately confer permanent immunity, Khan and Greenhalgh [17] introduced a time delay in the vaccination term. The maturation delay, which is the time taken for those 
immature exiting from the immature population and entering the mature population, was considered in [18, 19]. Since the new-born individuals have no ability to move freely, it takes a period of time to grow themselves and then move freely. Therefore, the individuals can contact with infected individuals over a period of time after they were born. Such a time delay which is called the freely-moving delay was considered in [20, 21], and Hopf bifurcation induced by such a delay was also studied therein. The distributed delay was considered [22], and the global asymptotic stability of disease free equilibrium and the endemic equilibrium were studied. The results [15-17, 19 21] suggest that delays can cause the loss of stability, and lead to various oscillations or periodic solutions.

It has been commonly accepted that some diseases are only spread or have more opportunities to be spread among children or immature individuals, such as mumps, measles, and chickenpox. Thus, it is necessary to incorporate stage structure into epidemic models. Aiello and Freedman [23] proposed and studied the well-known single species model with time-delayed stage structure. They showed the globally asymptotical stability of the positive equilibrium, and considered the effect of delay on the populations. After their work, different kinds of stage-structured models were studied [24, 25]. In these papers, they divided the species into two life stages: the mature stage and the immature stage, denoted by $y(t)$ and $x(t)$ respectively. The age to maturity was represented by a time delay $\tau$, which leaded to systems of retarded functional differential equations. $\alpha e^{-d \tau} y(t-\tau)$ represented the immature population born at time $t-\tau$ (with the mature birth rate $\alpha$ ) that survive to time $t$ (with the immature death rate $d$ ). For now, there have been a few authors dealing with population models with disease. For example, predator-prey system with disease in the prey was considered in [26, 27]. Hsieh and Hsiao [28] discussed a predator-prey model with disease infection in both populations. There are also a few papers studying on the epidemic models with stage structure [20, 21, 29].

Xiao and Chen [20] divided the population into two stages in an epidemic model: immature stage and mature stage, and assumed that disease transmission occurs only in the immature stage. $S(t)$ and $I(t)$ represent the population of susceptible and infected immature individuals, and $y(t)$ represents the population of mature individuals. Assume that the immature individuals take a period of time $\tau$ to maturity. For some species, such as oranguten and mamalian, they take a period of time $\omega$ to move freely after they are born. Thus, those immature born at time $t-\omega$, can contact with infected individuals at time $t$. 
By the meanings of $\tau$ and $\omega$, we know that $\tau>\omega$. They considered an SIS epidemic model with stage structure and a delay in the following form

$$
\left\{\begin{array}{l}
\frac{d S}{d t}=\alpha y(t)-d S(t)-\alpha e^{-d \tau} y(t-\tau)-\mu S(t-\omega) I(t)+\gamma I(t) \\
\frac{d I}{d t}=\mu S(t-\omega) I(t)-d I(t)-\gamma I(t) \\
\frac{d y}{d t}=\alpha e^{-d \tau} y(t-\tau)-\beta y^{2}(t)
\end{array}\right.
$$

where $\alpha$ is the natural birth rate, $d$ is the natural death rate of the immature stage, $\beta$ is the death rate of the mature individuals of logistic nature, $\mu$ is the disease transmission rate, and $\gamma$ is the recovery rate. It is found in [20] that the maturation delay has no effect on the dynamics of epidemic model and Hopf bifurcation can occur as the freely-moving delay increases.

Since individuals move around in space and the environment is usually inhomogeneous, spatial structure certainly has effect on disease transmission. It has turned out that introducing spatial structure into an epidemic model would reflect the reality better than the temporal models [30 35], when transmission mechanisms and control measures involve spatial movements. There have been many researchers focus on the effect of delay on the diffusive epidemic systems [36, 37], but the results of bifurcation behaviors are rare. we study in this paper the dynamics of epidemic system both in space and time, which can enhance the understanding of the epidemiological features of diseases.

According to the previous discussion, in fact, we can establish a reaction-diffusion system arising from modeling spatial spread of infectious diseases. Suppose that a species lives in an open bounded region $\Omega$ with smooth boundary $\partial \Omega$, and no individuals enter or leave the region at the boundary, i.e., the no-flux boundary condition. Adding random diffusion of susceptible, infected and mature individuals into (1), we have the following model 


$$
\left\{\begin{aligned}
\frac{\partial S(x, t)}{\partial t}= & d_{1} \triangle S(x, t)+\alpha y(x, t)-d S(x, t)-\alpha e^{-d \tau} y(x, t-\tau) \\
& -\mu S(x, t-\omega) I(x, t)+\gamma I(x, t), \\
\frac{\partial I(x, t)}{\partial t}= & d_{2} \triangle I(x, t)+\mu S(x, t-\omega) I(x, t)-d I(x, t)-\gamma I(x, t), \\
\frac{\partial y(x, t)}{\partial t}= & d_{3} \triangle y(x, t)+\alpha e^{-d \tau} y(x, t-\tau)-\beta y^{2}(x, t), \quad x \in \Omega, t>0, \\
\frac{\partial S(x, t)}{\partial \vec{n}}= & 0, \quad \frac{\partial I(x, t)}{\partial \vec{n}}=0, \quad \frac{\partial y(x, t)}{\partial \vec{n}}=0, \quad x \in \partial \Omega, t>0, \quad \\
S(x, \theta)= & \phi_{1}(x, \theta) \geq 0, I(x, \theta)=\phi_{2}(x, \theta) \geq 0, y(x, \theta)=\phi_{3}(x, \theta) \geq 0, \quad(x, \theta) \in \bar{\Omega} \times[-\tau, 0],
\end{aligned}\right.
$$

where the newly introduced coefficients $d_{1}, d_{2}, d_{3}$ denote the diffusion capabilities of susceptible, infected and mature individuals, respectively. In this paper, we do not limit the values of the diffusion coefficients. In fact, for some diseases, the infected individuals may reduce outside activity, thus the diffusion coefficient $d_{2}$ may be less than $d_{1}$ and $d_{3}$. However, for some diseases, such as rabies, the infected species may run much faster than the healthy ones, so $d_{2}$ may be much larger than the rest of diffusion coefficients. $\vec{n}$ is the outward unit normal vector on $\partial \Omega$. Recall that $\omega<\tau$, so we define the initial functions on $[-\tau, 0]$ for partial functional differential equation (2) [38].

Throughout the paper, without loss of generality, we consider the domain $\Omega=(0, l \pi)$, $l>0$. We use one-dimensional space for two reasons: on one hand, this is the case we can easily compute the eigenfunctions of the Laplacian; on the other, one dimension space has some biological interpretations, for example, the radius, the altitude, a long river, or depth of the water, etc. Assume $\phi_{1}, \phi_{2}, \phi_{3} \in \mathcal{C}=C([-\tau, 0], X)$, and endow the space

$$
X=\left\{\left(X_{1}, X_{2}, X_{3}\right)^{T}: X_{1}, X_{2}, X_{3} \in H^{2}(0, l \pi), \frac{\partial X_{1}}{\partial x}=\frac{\partial X_{2}}{\partial x}=\frac{\partial X_{3}}{\partial x}=0, x=0, l \pi\right\}
$$

with the regular $L^{2}$-inner product $\langle\cdot, \cdot\rangle$.

For reaction-diffusion systems, people usually focus on the existence of the stationary solutions first, including spatially homogeneous and spatially inhomogeneous solutions. The existence of spatially inhomogeneous steady state is difficult to analyze usually. They can be induced by the Turing bifurcation, which brings spatial inhomogeneity to the system by varying the diffusion rates. Meanwhile, we also care about the temporal development of the epidemic, especially Hopf bifurcation induced by time delay. For now, there have been some significant results on the spatio-temporal behaviors. Among them, three classes 
of problems have been investigated. Diffusion induced Turing instability has been studied in [39]. Time delay can induce Hopf bifurcation, which usually brings stable, spatially homogeneous oscillations [40, 41]. The third case is that the delay and diffusion can induce spatially inhomogeneous Hopf-Turing bifurcation, which is of codimension-2, and both spatial and temporal oscillations can be observed in this situation [42, 43]. In this paper, by detailed analysis on Hopf bifurcation, we find that Hopf bifurcation can induce both spatially homogenous and spatially inhomogeneous oscillations, and these oscillations are induced completely by the time delay and diffusion in the absence of Turing bifurcations, which means that the bifurcation we discussed is completely of codimension-one, and induce both spatial and temporal inhomogeneity.

The main object of this paper is to investigate the effect of the delay and diffusion on the dynamics of system (2). Using the freely-moving delay as the bifurcation parameter, we show that this delay can destabilize the positive constant equilibrium, and stable spatially homogeneous and inhomogeneous Hopf bifurcating periodic solutions occur near the first critical bifurcation value. Again, spatial inhomogeneity usually comes out near a HopfTuring bifurcation point [42]. However, our work is just about Hopf bifurcation inducing spatial oscillations. This is because all previous results indicate that the first Hopf bifurcation occurs when the corresponding eigenfunction of Laplacian is 1, in case of the Neumann boundary condition [40, 41]. In a system with Dirichlet boundary condition, it turns out that there are spatially inhomogeneous oscillations [44, 45]. However, this is not the case in the current paper with Neumann boundary condition. The results in [46] show that spatially inhomogeneous periodic solution can be stable only in the corresponding center manifold, implying that generically the model can only allow transient oscillatory patterns with spatial inhomogeneity. To our best knowledge, there is no result about Hopf bifurcation inducing stable inhomogeneous periodic solutions at the first critical value. We find a relation between the first bifurcation value and the diffusion coefficient, thus the first Hopf bifurcation occurs with a non-trivial eigenfunction, and spatial inhomogeneity appears. Moreover, in this paper, we find that diffusion induces several kinds of spatio-temporal behaviors with different spatial profiles.

About Hopf bifurcation in reaction-diffusion equations with time delays, many authors have worked on the normal form derivation to obtain the property of bifurcation [46-49]. The general approach is based on the center manifold reduction technique. By writing 
the system into an abstract ODE in an appropriate phase space, the derivations can be proceeded like those have been done in [50]. As all the derivations are restricted on a local center manifold, we pay the most attention on the first branch of Hopf bifurcation, since the rest ones must consist of unstable oscillations near the bifurcation values. According to [38], the corresponding eigenfunction of Laplacian determines the shape of bifurcating solutions. Thus, in this paper, we make a great effort to investigate the relation among the first bifurcation value, the corresponding eigenfunction, and the diffusion coefficients, then try to find out how diffusion induces different kinds of stable, spatially inhomogeneous oscillations.

This paper is structured as follows: In the next section, we discuss the stability of the positive constant equilibrium and the existence of Hopf bifurcation. In Section 3, by using the normal form theory and the center manifold reduction, we determine the stability of the spatially bifurcating periodic solutions and the direction of the Hopf bifurcations. In Section 4. we investigate the effect of diffusion on the first Hopf bifurcation value and on the dynamics of the system, then the conditions for the appearance of stable spatially inhomogeneous oscillation are determined. In Section 5, we present some numerical simulations to support our theoretical analysis. The paper ends with conclusion and discussion in Section 6 .

\section{EQUILIBRIA, THEIR STABILITY AND THE EXISTENCE OF HOPF BIFUR- CATIONS}

The very first thing to analyze the dynamics of system (2) is to study the existence of equilibria, then by finding the stability boundary we can detect some possible bifurcation behaviors. In this section we mainly use time delays and diffusion rates as bifurcation parameters.

In fact, system (2) always has the following nonnegative constant equilibria: a trivial equilibrium $E_{0}(0,0,0)$ and a semi-trivial disease-free constant equilibrium $E_{1}(\bar{S}, 0, \bar{y})$, where

$$
\bar{S}=\frac{\alpha^{2} e^{-d \tau}\left(1-e^{-d \tau}\right)}{d \beta}, \bar{y}=\frac{\alpha e^{-d \tau}}{\beta}
$$

Moreover, denote the basic reproduction ratio by

$$
R_{0}=\frac{\mu \alpha^{2} e^{-d \tau}\left(1-e^{-d \tau}\right)}{d \beta(d+\gamma)}
$$


If $R_{0}>1$, then (2) has a positive constant equilibrium $E_{2}\left(S^{*}, I^{*}, y^{*}\right)$, where

$$
S^{*}=\frac{d+\gamma}{\mu}, I^{*}=\frac{(d+\gamma)}{\mu}\left(R_{0}-1\right), y^{*}=\frac{\alpha e^{-d \tau}}{\beta} .
$$

\subsection{The stability analysis of the trivial equilibrium and the disease-free equilib-} rium

In order to investigate the local stability we write the linearization of system (2) at the trivial equilibrium $E_{0}(0,0,0)$ as

$$
\left\{\begin{array}{l}
\frac{\partial S(x, t)}{\partial t}=d_{1} \triangle S(x, t)+\alpha y(x, t)-d S(x, t)-\alpha e^{-d \tau} y(x, t-\tau)+\gamma I(x, t), \\
\frac{\partial I(x, t)}{\partial t}=d_{2} \triangle I(x, t)-d I(x, t)-\gamma I(x, t) \\
\frac{\partial y(x, t)}{\partial t}=d_{3} \triangle y(x, t)+\alpha e^{-d \tau} y(x, t-\tau) .
\end{array}\right.
$$

From $\mathrm{Wu}$ [38], obviously, the eigenfunctions of $\triangle$ on $[0, l \pi]$ are $\cos \frac{n}{l} x, n=0,1,2 \ldots$ The characteristic equation of (3) can be obtained by plugging

$$
\left\{\begin{array}{l}
S(x, t)=\sum_{n} \cos \frac{n x}{l} S_{n}(t), \\
I(x, t)=\sum_{n}^{n} \cos \frac{n x}{l} I_{n}(t) \\
y(x, t)=\sum_{n}^{n} \cos \frac{n x}{l} y_{n}(t)
\end{array}\right.
$$

into (3), after which a sequence of ODEs are obtained. Hence we have a sequence of characteristic equations,

$$
\left(\lambda+d+d_{1} \frac{n^{2}}{l^{2}}\right)\left(\lambda+d+\gamma+d_{2} \frac{n^{2}}{l^{2}}\right)\left(\lambda-\alpha e^{-d \tau} e^{-\lambda \tau}+d_{3} \frac{n^{2}}{l^{2}}\right)=0
$$

where $n \in \mathbb{N}_{0}=\mathbb{N} \cup\{0\}$. Clearly, we have some roots $\lambda_{1, n}=-d-d_{1} \frac{n^{2}}{l^{2}}<0$ and $\lambda_{2, n}=$ $-d-\gamma-d_{2} \frac{n^{2}}{l^{2}}<0$. The rest roots of $(4)$ are given by solving the following equation

$$
\lambda-\alpha e^{-d \tau} e^{-\lambda \tau}+d_{3} \frac{n^{2}}{l^{2}}=0 .
$$

Let

$$
g_{1}(\lambda)=\lambda-\alpha e^{-d \tau} e^{-\lambda \tau}+d_{3} \frac{n^{2}}{l^{2}}
$$

It is easy to show that $\left.g_{1}(0)\right|_{n=0}=-\alpha e^{-d \tau}<0, \lim _{\lambda \rightarrow+\infty} g_{1}(\lambda)=+\infty$. Hence 5 has at least one positive root. Therefore, $E_{0}$ is always unstable. 
Similarly, the characteristic equations associated with the linearization of system (2) at the equilibrium $E_{1}$ are of the form

$$
\left(\lambda+d+d_{1} \frac{n^{2}}{l^{2}}\right)\left(\lambda-\mu \bar{S}+d+\gamma+d_{2} \frac{n^{2}}{l^{2}}\right)\left(\lambda-\alpha e^{-d \tau} e^{-\lambda \tau}+2 \beta \bar{y}+d_{3} \frac{n^{2}}{l^{2}}\right)=0
$$

with $n \in \mathbb{N}_{0}$.

To investigate the location of the roots, we first consider the third term, i.e., the following equation

$$
\lambda-\alpha e^{-d \tau} e^{-\lambda \tau}+2 \beta \bar{y}+d_{3} \frac{n^{2}}{l^{2}}=0 .
$$

Lemma 1. All roots of Eq. (7) have negative real part.

Proof. When $\tau=0$, we have $\lambda=\alpha-2 \alpha-d_{3} \frac{n^{2}}{l^{2}}=-\alpha-d_{3} \frac{n^{2}}{l^{2}}<0$, thus $\lambda=0$ is not a root. Suppose that $\lambda=i \sigma(\sigma>0)$ is a root of Eq. (7), then we have

$$
i \sigma-\alpha e^{-d \tau}(\cos \sigma \tau-i \sin \sigma \tau)+2 \beta \bar{y}+d_{3} \frac{n^{2}}{l^{2}}=0
$$

Separating the real and imaginary parts, we get

$$
\sigma=-\alpha e^{-d \tau} \sin \sigma \tau, \quad 2 \beta \bar{y}+d_{3} \frac{n^{2}}{l^{2}}=\alpha e^{-d \tau} \cos \sigma \tau
$$

Adding up the squares of both equations of (8), we have

$$
\sigma^{2}+\left(2 \alpha e^{-d \tau}+d_{3} \frac{n^{2}}{l^{2}}\right)^{2}=\alpha^{2}\left(e^{-d \tau}\right)^{2}
$$

This is impossible. Then, by Li et al. [51], we have the conclusion.

The rest roots of (6) are $\lambda_{1, n}=-d-d_{1} \frac{n^{2}}{l^{2}}<0, \lambda_{2, n}=\mu \bar{S}-d-\gamma-d_{2} \frac{n^{2}}{l^{2}}$. It is easy to verify that when $R_{0}=\frac{\mu \bar{S}}{d+\gamma}<1, \lambda_{2, n}<0$ and when $R_{0}>1, \lambda_{2,0}=\mu \bar{S}-d-\gamma>0$.

From the previous discussion, we know that when $R_{0}<1$, the roots of (6) all have negative real part, and when $R_{0}>1$, Eq. (6) has roots with positive real part. Therefore, the disease free equilibrium $E_{1}$ is locally asymptotically stable when $R_{0}<1$, and it is unstable when $R_{0}>1$. In fact, one can prove that the critical condition $R_{0}=1$ corresponds to a transcritical bifurcation at $E_{1}$. 


\subsection{Stability of the positive equilibrium and Hopf bifurcation induced by delay}

The linearization of system (2) at the positive equilibrium $E_{2}\left(S^{*}, I^{*}, y^{*}\right)$ is

$$
\frac{\partial}{\partial t}\left(\begin{array}{c}
S(x, t) \\
I(x, t) \\
y(x, t)
\end{array}\right)=\left(D \triangle+B_{1}\right)\left(\begin{array}{c}
S(x, t) \\
I(x, t) \\
y(x, t)
\end{array}\right)+B_{2}\left(\begin{array}{c}
S(x, t-\omega) \\
I(x, t-\omega) \\
y(x, t-\omega)
\end{array}\right)+B_{3}\left(\begin{array}{c}
S(x, t-\tau) \\
I(x, t-\tau) \\
y(x, t-\tau)
\end{array}\right),
$$

where $D=\operatorname{diag}\left\{d_{1}, d_{2}, d_{3}\right\}$,

$$
B_{1}=\left(\begin{array}{ccc}
-d & -\mu S^{*}+\gamma & \alpha \\
0 & \mu S^{*}-d-\gamma & 0 \\
0 & 0 & -2 \beta y^{*}
\end{array}\right), B_{2}=\left(\begin{array}{ccc}
-\mu I^{*} & 0 & 0 \\
\mu I^{*} & 0 & 0 \\
0 & 0 & 0
\end{array}\right), B_{3}=\left(\begin{array}{ccc}
0 & 0 & -\alpha e^{-d \tau} \\
0 & 0 & 0 \\
0 & 0 & \alpha e^{-d \tau}
\end{array}\right) .
$$

The characteristic equation of $(9)$ is

$$
\operatorname{det}\left(\lambda I_{3}-M_{n}-B_{1}-B_{2} e^{-\lambda \omega}-B_{3} e^{-\lambda \tau}\right)=0,
$$

where $I_{3}$ is the $3 \times 3$ identity matrix and $M_{n}=-\frac{n^{2}}{l^{2}} D, n \in \mathbb{N}_{0}$. That is, each eigenvalue $\lambda$ is a root of the following equation

$$
\left(\lambda-\alpha e^{-d \tau} e^{-\lambda \tau}+2 \beta y^{*}+d_{3} \frac{n^{2}}{l^{2}}\right)\left[\lambda^{2}+A_{n} \lambda+B_{n}+e^{-\lambda \omega}\left(C \lambda+D_{n}\right)\right]=0
$$

with $n \in \mathbb{N}_{0}$,

$$
\begin{aligned}
& A_{n}=d+d_{1} \frac{n^{2}}{l^{2}}+d_{2} \frac{n^{2}}{l^{2}}, \\
& B_{n}=d_{2} \frac{n^{2}}{l^{2}}\left(d+d_{1} \frac{n^{2}}{l^{2}}\right), \\
& C=\mu I^{*},
\end{aligned}
$$

and

$$
D_{n}=\mu I^{*} d_{2} \frac{n^{2}}{l^{2}}+\mu I^{*} d .
$$

Clearly, $\lambda=0$ is not a root of (11), which excludes the existence of Turing bifurcation. By Lemma 1, we know that the roots of $\lambda-\alpha e^{-d \tau} e^{-\lambda \tau}+2 \beta y^{*}+d_{3} \frac{n^{2}}{l^{2}}=0$ have negative real part. Then, it remains to consider the roots of the following equation

$$
\lambda^{2}+A_{n} \lambda+B_{n}+e^{-\lambda \omega}\left(C \lambda+D_{n}\right)=0 .
$$

When $\omega=0$, Eq. 12 becomes the following sequence of quadratic polynomial equations

$$
\lambda^{2}+\left(A_{n}+C\right) \lambda+\left(B_{n}+D_{n}\right)=0, \quad n \in \mathbb{N}_{0},
$$


where

$$
\begin{aligned}
& A_{n}+C=d+d_{1} \frac{n^{2}}{l^{2}}+d_{2} \frac{n^{2}}{l^{2}}+\mu I^{*}>0, \\
& B_{n}+D_{n}=d_{2} \frac{n^{2}}{l^{2}}\left(d+d_{1} \frac{n^{2}}{l^{2}}\right)+\mu I^{*} d_{2} \frac{n^{2}}{l^{2}}+\mu I^{*} d>0 .
\end{aligned}
$$

We know that all roots of Eq. (13) have negative real part. Therefore, when $R_{0}>1$, all the roots of Eq. (11) have negative real part for $\omega=0$.

Next we should seek critical values of $\omega$ such that there exists a pair of simple purely imaginary eigenvalues, which may lead to Hopf bifurcations. Assume that $i z(z>0)$ is a root of Eq. (12). Then we obtain

$$
-z^{2}+i z A_{n}+B_{n}+(\cos \omega z-i \sin \omega z)\left(i z C+D_{n}\right)=0
$$

Separating the real and imaginary parts, we have

$$
\left\{\begin{array}{l}
-z^{2}+B_{n}=-C z \sin \omega z-D_{n} \cos \omega z \\
A_{n} z=-C z \cos \omega z+D_{n} \sin \omega z
\end{array}\right.
$$

Squaring and adding both equations of (15) lead to

$$
z^{4}+\left(A_{n}^{2}-2 B_{n}-C^{2}\right) z^{2}+B_{n}^{2}-D_{n}^{2}=0
$$

where

$$
\begin{aligned}
& A_{n}^{2}-2 B_{n}-C^{2}=\left(d_{2} \frac{n^{2}}{l^{2}}\right)^{2}+\left(d+d_{1} \frac{n^{2}}{l^{2}}+\mu I^{*}\right)\left(d+d_{1} \frac{n^{2}}{l^{2}}-\mu I^{*}\right), \\
& B_{n}^{2}-D_{n}^{2}=\left(B_{n}+D_{n}\right)\left(B_{n}-D_{n}\right) .
\end{aligned}
$$

Noticing that $B_{n}+D_{n}>0$, the sign of $B_{n}^{2}-D_{n}^{2}$ coincides with that of $B_{n}-D_{n}$, where

$$
\begin{aligned}
B_{n}-D_{n} & =d_{2} \frac{n^{2}}{l^{2}}\left(d+d_{1} \frac{n^{2}}{l^{2}}\right)-\mu I^{*} d_{2} \frac{n^{2}}{l^{2}}-\mu I^{*} d \\
& =d_{1} d_{2} \frac{1}{l^{4}} n^{4}+\left(d-\mu I^{*}\right) d_{2} \frac{1}{l^{2}} n^{2}-\mu I^{*} d
\end{aligned}
$$

Since $B_{0}-D_{0}=-\mu I^{*} d<0$ and $B_{n}-D_{n}$ is a quadratic polynomial with respect to $n^{2}$, we can conclude by 18 that there exists $n_{1} \in \mathbb{N}_{0}$, such that

$$
\begin{array}{ll}
B_{n}-D_{n}<0 & \text { for } 0 \leq n \leq n_{1}, \\
B_{n}-D_{n}>0 & \text { for } n \geq n_{1}+1, n \in \mathbb{N}_{0} .
\end{array}
$$

Denote the positive real root of the equation $B_{n}-D_{n}=0$ by $n_{2}\left(n_{1}<n_{2}<n_{1}+1\right)$, then we have

$$
B_{n_{2}}-D_{n_{2}}=d_{1} d_{2} \frac{1}{l^{4}} n_{2}^{4}+\left(d-\mu I^{*}\right) d_{2} \frac{1}{l^{2}} n_{2}^{2}-\mu I^{*} d=0
$$


Since $-\mu I^{*} d<0$, we have $d_{1} d_{2} \frac{1}{l^{4}} n_{2}^{4}+\left(d-\mu I^{*}\right) d_{2} \frac{1}{l^{2}} n_{2}^{2}=\left(d+d_{1} \frac{n_{2}^{2}}{l^{2}}-\mu I^{*}\right) d_{2} \frac{1}{l^{2}} n_{2}^{2}>0$. It means that

$$
d+d_{1} \frac{n_{2}^{2}}{l^{2}}-\mu I^{*}>0
$$

By (17), we have

$$
A_{n_{2}}^{2}-2 B_{n_{2}}-C^{2}=\left(d_{2} \frac{n_{2}^{2}}{l^{2}}\right)^{2}+\left(d+d_{1} \frac{n_{2}^{2}}{l^{2}}+\mu I^{*}\right)\left(d+d_{1} \frac{n_{2}^{2}}{l^{2}}-\mu I^{*}\right)>0 .
$$

Noticing $n_{1}+1>n_{2}$ and by (19) and $(22)$, we get

$$
A_{n}^{2}-2 B_{n}-C^{2}>0, \text { for } n \geq n_{1}+1, n_{1} \in \mathbb{N}_{0}
$$

From (19) and (23), we can conclude that for $n \in \mathbb{N}_{0}$ and $n \leq n_{1}$, (16) has only one positive real root $z_{n}$, where

$$
z_{n}=\sqrt{\frac{-\left(A_{n}^{2}-2 B_{n}-C^{2}\right)+\sqrt{\triangle}}{2}}
$$

with

$$
\triangle=\left(A_{n}^{2}-2 B_{n}-C^{2}\right)^{2}-4\left(B_{n}^{2}-D_{n}^{2}\right) .
$$

For $n \in \mathbb{N}_{0}$ and $n \geq n_{1}+1$, 16 has no positive real roots.

According to the above discussion, the following results on Eq. 12 follow immediately.

Lemma 2. Suppose that $R_{0}>1$, and $n_{1}$ and $z_{n}$ are defined by (19) and (24), respectively. Then Eq. (12) has a pair of purely imaginary roots $\pm i z_{n}$ for each $n \in\left\{0,1, \ldots, n_{1}\right\}$ and has no purely imaginary roots for $n \geq n_{1}+1, n \in \mathbb{N}_{0}$.

Now we calculate the critical Hopf bifurcation value $\omega$. By 15 , we have

$$
\begin{aligned}
& \sin z_{n} \omega=\frac{A_{n} z_{n} D_{n}-\left(B_{n}-z_{n}^{2}\right) C z_{n}}{\left(C z_{n}\right)^{2}+D_{n}^{2}}=S_{n}\left(z_{n}\right), \\
& \cos z_{n} \omega=-\frac{A_{n} C z_{n}^{2}+\left(B_{n}-z_{n}^{2}\right) D_{n}}{D_{n}^{2}+\left(C z_{n}\right)^{2}}=C_{n}\left(z_{n}\right) .
\end{aligned}
$$

For $n \in\left\{0,1, \ldots, n_{1}\right\}$, define

$$
\omega_{n}^{j}= \begin{cases}\frac{1}{z_{n}}\left(\arccos C_{n}\left(z_{n}\right)+2 j \pi\right), & \text { if } S_{n} \geq 0 \\ \frac{1}{z_{n}}\left(2 \pi-\arccos C_{n}\left(z_{n}\right)+2 j \pi\right), & \text { if } S_{n}<0\end{cases}
$$

In fact,

$$
S_{n}\left(z_{n}\right)=\frac{A_{n} z_{n} D_{n}-\left(B_{n}-z_{n}^{2}\right) C z_{n}}{\left(C z_{n}\right)^{2}+D_{n}^{2}}=\frac{z_{n}\left[A_{n} D_{n}-B_{n} C+z_{n}^{2} C\right]}{\left(C z_{n}\right)^{2}+D_{n}^{2}}
$$


where

$$
\begin{aligned}
& A_{n} D_{n}-B_{n} C \\
& =\left(d+d_{1} \frac{n^{2}}{l^{2}}+d_{2} \frac{n^{2}}{l^{2}}\right)\left(\mu I^{*} d_{2} \frac{n^{2}}{l^{2}}+\mu I^{*} d\right)-\left(d_{2} \frac{n^{2}}{l^{2}}\left(d+d_{1} \frac{n^{2}}{l^{2}}\right)\right) \mu I^{*} \\
& =\mu I^{*}\left[d\left(d+d_{1} \frac{n^{2}}{l^{2}}+d_{2} \frac{n^{2}}{l^{2}}\right)+\left(d_{2} \frac{n^{2}}{l^{2}}\right)^{2}\right]>0 .
\end{aligned}
$$

Thus, when $n \in\left\{0,1, \ldots, n_{1}\right\}, S_{n} \geq 0$, we have

$$
\omega_{n}^{j}=\frac{1}{z_{n}}\left[\arccos C_{n}\left(z_{n}\right)+2 j \pi\right] .
$$

Define the very first critical value as

$$
\omega^{*}=\omega_{n_{0}}^{0}=\min _{n \in\left\{0,1, \ldots, n_{1}\right\}}\left\{\omega_{n}^{0}\right\}, \quad z^{*}=z_{n_{0}} .
$$

To ensure the existence of Hopf bifurcation, we still need to verify the following transversality condition.

Lemma 3. Suppose $R_{0}>1$, then for $n \in\left\{0,1, \ldots, n_{1}\right\}$ and $j \in \mathbb{N}_{0}$, $\left.\frac{\mathrm{dRe} \lambda(\omega)}{\mathrm{d} \omega}\right|_{\omega=\omega_{n}^{j}}>0$.

Proof. Differentiating the two sides of Eq. 12 with respective to $\omega$, we obtain

$$
\left(\frac{\mathrm{d} \lambda}{\mathrm{d} \omega}\right)^{-1}=\frac{2 \lambda+A_{n}}{e^{-\lambda \omega}\left(C \lambda^{2}+D_{n} \lambda\right)}+\frac{C-D_{n} \omega-\omega C \lambda}{\lambda\left(C \lambda+D_{n}\right)}
$$

Using (12) and (15), we obtain

$$
\begin{aligned}
& \left.\operatorname{Re}\left(\frac{\mathrm{d} \lambda}{\mathrm{d} \omega}\right)^{-1}\right|_{\omega=\omega_{n}^{j}} \\
& \left.=\operatorname{Re}\left[\frac{2 \lambda+A_{n}}{-\lambda\left(\lambda^{2}+A_{n} \lambda+B_{n}\right)}\right]_{\omega=\omega_{n}^{j}}+\operatorname{Re}\left[\frac{C-D_{n} \omega-\omega C \lambda}{\lambda\left(C \lambda+D_{n}\right)}\right]_{\omega=\omega_{n}^{j}}\right]_{n}+\operatorname{Re}\left[\frac{C-D_{n} \omega-i z_{n} \omega C}{i z_{n}\left(i z_{n} C+A_{n}\right)}\right]_{\omega=\omega_{n}^{j}} \\
& =\operatorname{Re}\left[\frac{1}{-i z_{n}\left(-z_{n}^{2}+i z_{n} A_{n}+B_{n}\right)}\right]_{\omega=\omega_{n}^{j}} \\
& =\frac{1}{z_{n}} \frac{z_{n} A_{n}^{2}-2 z_{n}\left(B_{n}-z_{n}^{2}\right)}{z_{n}^{2} A_{n}^{2}+\left(B_{n}-z_{n}^{2}\right)^{2}}+\frac{1}{z_{n}} \frac{-C^{2} z_{n}}{C^{2} z_{n}^{2}+D_{n}^{2}} \\
& =\frac{2 z_{n}^{2}+\left(A_{n}^{2}-2 B_{n}-C^{2}\right)}{C^{2} z_{n}^{2}+D_{n}^{2}} \\
& =\frac{\sqrt{\left(A_{n}^{2}-2 B_{n}-C^{2}\right)^{2}-4\left(B_{n}^{2}-D_{n}^{2}\right)}}{C^{2} z_{n}^{2}+D_{n}^{2}}>0 .
\end{aligned}
$$

The proof is complete.

According to the above discussion and corollary 2.4 of Ruan and Wei [52], we know that the roots of (11) have negative real part when $0 \leq \omega<\omega^{*}$, and (11) has a pair of simple pure imaginary roots when $\omega=\omega_{n}^{j}$. Moreover, (11) has at least one pair of conjugate complex roots with positive real part when $\omega>\omega^{*}$. Due to the general Hopf bifurcation theorem [38, 47], we have the following theorem. 
Theorem 1. Suppose $R_{0}>1$.

(1) The equilibrium $E_{2}$ of system (2) is locally asymptotically stable for $0 \leq \omega<\omega^{*}$ and is unstable for $\omega>\omega^{*}$.

(2) System (2) undergoes a Hopf bifurcation at the equilibrium $E_{2}$ when $\omega=\omega_{n}^{j}$, for $j \in \mathbb{N}_{0}$ and $n \in\left\{0,1, \ldots, n_{1}\right\}$.

Generally, Hopf bifurcation leads a system to oscillations in the time-direction. However, to get the stability of the oscillation and to know where it happens, we need to calculate the normal forms, which will be completed in the coming section.

\section{DIRECTION AND STABILITY OF SPATIALLY HOPF BIFURCATION}

From Theorem 1, we know that system (2) undergoes Hopf bifurcations at the equilibrium $E_{2}$ when $\omega=\omega_{n}^{j}$. In this section, we investigate the direction and stability of the bifurcating periodic solutions by using the center manifold theorem and the normal form theory of partial functional differential equations [38, 47]. Obviously, according to Theorem 1 and the results in [38, only the periodic solutions bifurcating from the first Hopf bifurcation point $\omega^{*}$ could be stable, because at all the rest bifurcation points, the system have unstable manifold. Thus in this section we only consider the properties of periodic solutions near the first Hopf bifurcation point $\omega^{*}$.

Let $u_{1}(x, t)=S(x, \omega t)-S^{*}, u_{2}(x, t)=I(x, \omega t)-I^{*}, u_{3}(x, t)=y(x, \omega t)-y^{*}$, then system (2) can be transformed into

$$
\left\{\begin{aligned}
\frac{\partial u_{1}}{\partial t}= & \omega\left[d_{1} \triangle u_{1}(x, t)+\alpha u_{3}(x, t)-d u_{1}(x, t)-\alpha e^{-d \tau} u_{3}\left(x, t-\tau^{*}\right)\right. \\
& \left.-\mu I^{*} u_{1}(x, t-1)-\mu S^{*} u_{2}(x, t)+\gamma u_{2}(x, t)-\mu u_{1}(x, t-1) u_{2}(x, t)\right], \\
\frac{\partial u_{2}}{\partial t}= & \omega\left[d_{2} \triangle u_{2}(x, t)+\mu I^{*} u_{1}(x, t-1)+\mu S^{*} u_{2}(x, t)-d u_{2}(x, t)-\gamma u_{2}(x, t)\right. \\
& \left.+\mu u_{1}(x, t-1) u_{2}(x, t)\right], \\
\frac{\partial u_{3}}{\partial t}= & \omega\left[d_{3} \triangle u_{3}(x, t)+\alpha e^{-d \tau} u_{3}\left(x, t-\tau^{*}\right)-2 \beta y^{*} u_{3}(x, t)-\beta u_{3}^{2}(x, t)\right],
\end{aligned}\right.
$$

where $\tau^{*}=\tau / \omega$. Setting $U(t)=\left(u_{1}(x, t), u_{2}(x, t), u_{3}(x, t)\right)^{T}$, then in the abstract space $\mathcal{C}=C\left(\left[-\tau^{*}, 0\right], X\right),(28)$ can be rewritten as

$$
\frac{d U}{d t}=\omega D \triangle U(t)+L(\omega)\left(U_{t}\right)+f\left(U_{t}, \omega\right)
$$


where $\operatorname{dom}(D \triangle)=\left\{\left(u_{1}, u_{2}, u_{3}\right)^{T}: u_{1}, u_{2}, u_{3} \in H^{2}(0, l \pi), u_{1 x}=u_{2 x}=u_{3 x}=0\right.$, at $\left.x=0, l \pi\right\}$ and $L(\omega)(\cdot): \mathcal{C} \rightarrow X, f: \mathcal{C} \times \mathbb{R} \rightarrow X$ are given respectively by

$$
\begin{aligned}
& \begin{aligned}
L(\omega)(\phi) & =\omega\left[B_{1} \phi(0)+B_{2} \phi(-1)+B_{3} \phi\left(-\tau^{*}\right)\right], \\
f(\phi, \omega) & =\omega\left(f_{1}(\phi, \omega), f_{2}(\phi, \omega), f_{3}(\phi, \omega)\right)^{T} \\
& =\omega\left(-\mu \phi_{1}(-1) \phi_{2}(0), \mu \phi_{1}(-1) \phi_{2}(0),-\beta \phi_{3}^{2}(0)\right)^{T} .
\end{aligned}
\end{aligned}
$$

where

$$
\phi=\left(\phi_{1}, \phi_{2}, \phi_{3}\right)^{T} \in \mathcal{C} .
$$

Let $\omega=\omega^{*}+\nu$, with small parameter $\nu \in \mathbb{R}$, then Eq. (29) can be written as

$$
\frac{d U}{d t}=\omega^{*} D \triangle U(t)+L\left(\omega^{*}\right)\left(U_{t}\right)+F\left(U_{t}, \nu\right),
$$

where

$$
F(\phi, \nu)=\nu D \triangle \phi(0)+L(\nu)(\phi)+f\left(\phi, \omega^{*}+\nu\right), \quad \text { for } \phi \in \mathcal{C} .
$$

Consider the linearized system of (31)

$$
\frac{d U}{d t}=\omega^{*} D \triangle U(t)+L\left(\omega^{*}\right)\left(U_{t}\right)
$$

From the previous discussion, when $\nu=0$ (i.e. $\omega=\omega^{*}$ ), system (31) undergoes Hopf bifurcation at the equilibrium $(0,0,0)$. We can also get $\Lambda_{n}:=\left\{i z^{*} \omega^{*},-i z^{*} \omega^{*}\right\}$ are simple pure imaginary characteristic values of $(32)$ and obtain the linear functional differential equation

$$
\frac{d X}{d t}=-\omega^{*} D \frac{n^{2}}{l^{2}} X(t)+L\left(\omega^{*}\right)\left(X_{t}\right) .
$$

By the Riesz representation theorem, there exists a bounded variation function $\eta_{n}\left(\theta, \omega^{*}\right)$ $\left(-\tau^{*} \leq \theta \leq 0\right)$ such that

$$
-\omega^{*} D \frac{n^{2}}{l^{2}} \phi(0)+L\left(\omega^{*}\right)(\phi)=\int_{-\tau^{*}}^{0} d \eta_{n}(\theta, \omega) \phi(\theta)
$$

for $\phi \in C\left(\left[-\tau^{*}, 0\right], \mathbb{R}^{3}\right)$.

In fact, we can choose

$$
\eta_{n}\left(\theta, \omega^{*}\right)= \begin{cases}\omega^{*}\left(-D \frac{n^{2}}{l^{2}}+B_{1}+B_{2}\right), & \theta=0, \\ \omega^{*} B_{2}, & \theta \in(-1,0), \\ 0, & \theta \in\left(-\tau^{*},-1\right], \\ -\omega^{*} B_{3}, & \theta=-\tau^{*} .\end{cases}
$$


Let $A\left(\omega^{*}\right)$ denote the infinitesimal generator of the semigroup induced by the solutions of Eq. (33) and $A^{*}$ denote the formal adjoint of $A\left(\omega^{*}\right)$ under the bilinear form

$$
(\psi(s), \phi(\theta))=\psi(0) \phi(0)-\int_{-\tau^{*}}^{0} \int_{\xi=0}^{\theta} \psi(\xi-\theta) d \eta_{n}\left(\theta, \omega^{*}\right) \phi(\xi) d \xi,
$$

for $\phi \in C\left(\left[-\tau^{*}, 0\right], \mathbb{R}^{3}\right), \psi \in C^{1}\left(\left[0, \tau^{*}\right], \mathbb{R}^{3^{T}}\right)$.

To determine the direction of Hopf bifurcation and the stability of the periodic solutions, we only need to compute the coefficients $\mu_{2}, \beta_{2}, T_{2}$ [48]. The calculations are very long, so we leave them in Appendix A.

Based on the derivation in Appendix A, we can compute each $g_{i j}$ in A11. Thus we can compute the following values:

$$
\begin{aligned}
& c_{1}(0)=\frac{i}{2 z^{*} \omega^{*}}\left(g_{11} g_{20}-2\left|g_{11}\right|^{2}-\frac{\left|g_{02}\right|^{2}}{3}\right)+\frac{g_{21}}{2}, \\
& \mu_{2}=-\frac{\operatorname{Re}\left(c_{1}(0)\right)}{\operatorname{Re}\left(\lambda^{\prime}\left(\omega^{*}\right)\right)}, \\
& \beta_{2}=2 \operatorname{Re}\left(c_{1}(0)\right), \\
& T_{2}=-\frac{\operatorname{Im}\left(c_{1}(0)\right)+\mu_{2} \operatorname{Im}\left(\lambda^{\prime}\left(\omega^{*}\right)\right)}{z^{*} \omega^{*}} .
\end{aligned}
$$

As a direct application of the results in [48, we know that $\mu_{2}$ determines the direction of the Hopf bifurcation: if $\mu_{2}>0\left(\mu_{2}<0\right)$, then the Hopf bifurcation is supercritical (subcritical) and the bifurcating periodic solutions exist for $\omega>\omega^{*}\left(\omega<\omega^{*}\right)$; and $\beta_{2}$ determines the stability of the bifurcating periodic solutions: the bifurcating periodic solutions are stable (unstable) if $\beta_{2}<0\left(\beta_{2}>0\right) ; T_{2}$ determines the period of the bifurcating periodic solutions: the period increases (decreases) if $T_{2}>0\left(T_{2}<0\right)$.

Since $\operatorname{Re}\left(\lambda^{\prime}\left(\omega^{*}\right)>0\right.$, we get the following conclusion near a neighborhood of $\omega^{*}$.

Theorem 2. If $\operatorname{Re}\left(c_{1}(0)\right)<0(>0)$, then $\mu_{2}>0(<0), \beta_{2}<0(>0)$, and the Hopf bifurcating periodic solutions exist for $\omega>\omega^{*}\left(<\omega^{*}\right)$ are orbitally asymptotically stable (unstable).

Remark 1. From Theorem 2.2 in Section 6.2 of [38], system (29) has a family of periodic solutions bifurcating from $(0,0,0)$ parameterized by small $\epsilon$. When $\nu=\nu(\epsilon)$ and $\epsilon$ are near 0, (i.e. when $\omega$ is near $\left.\omega^{*}\right)$, the periodic solutions have the following representations

$$
U_{t}(\nu, \theta)(x)=\epsilon \operatorname{Rep}_{1}(\theta) e^{i z^{*} \omega^{*} t} \cos \frac{n_{0}}{l} x+O\left(\epsilon^{2}\right) .
$$

Here we assume $\omega^{*}=\omega_{n_{0}}^{0}$. From Lemma 4, $p_{1}(\theta)=\left(1, \xi_{1}, \xi_{2}\right)^{T} e^{i z^{*} \omega^{*} \theta}$, where $\xi_{2}=0$. Thus, the third component $y(t)$ of system (2) has no spatial oscillations. 


\section{SPATIAL PROFILES OF THE FIRST BRANCH OF HOPF BIFURCATING SOLUTIONS}

From discussions in the previous two sections, we know that the first Hopf bifurcation point is the most important value among all the bifurcation values. In fact, from $\mathrm{Wu}$ [38] and Remark 1, only the first branch of Hopf bifurcating periodic solutions may be stable near the critical point, and the shape, or the spatial profile we say, of these periodic solutions depends on the corresponding eigenfunction $\cos \frac{n_{0}}{l} x$ of Laplacian. It means that if the very first bifurcation point $\omega^{*}=\omega_{0}^{0}$, i.e., $n_{0}=0$, then the corresponding eigenfunction is 1 , and the Hopf bifurcating periodic solutions are spatially homogeneous. If $\omega^{*}=\omega_{n_{0}}^{0}\left(n_{0} \neq 0\right)$, then the bifurcating periodic solutions are spatially inhomogeneous. Therefore, in this section, we will investigate when the first bifurcation value occurs to show the different kinds of spatial distributions.

Now, we investigate the relation among the first bifurcation value, the corresponding eigenfunction, and the diffusion coefficients.

Consider the first Hopf bifurcation value with two special cases: $d_{2} \rightarrow 0$ and $d_{2} \rightarrow+\infty$, respectively. We can simply obtain the following two results.

Theorem 3. Suppose $d_{2} \rightarrow 0$, then for $n \in\left\{0,1, \ldots, n_{1}\right\}, \omega_{n}^{0}$ takes the minimum value when $n=0$, that is, $\omega^{*}=\omega_{0}^{0}$.

\section{Proof.}

In fact, since $\sin z_{n} \omega_{n}^{0}>0$, we have

$$
\omega_{n}^{0}=\frac{1}{z_{n}}\left[\arccos C_{n}\left(z_{n}\right)\right]=\frac{\arccos \left[-\frac{A_{n} C z_{n}^{2}+\left(B_{n}-z_{n}^{2}\right) D_{n}}{D_{n}^{2}+\left(C z_{n}\right)^{2}}\right]}{\sqrt{\frac{-\left(A_{n}^{2}-2 B_{n}-C^{2}\right)+\sqrt{\triangle}}{2}}} .
$$

Denote by

$$
h_{1}(n)=2 z_{n}^{2}=-\left(A_{n}^{2}-2 B_{n}-C^{2}\right)+\sqrt{\triangle} .
$$

When $d_{2} \rightarrow 0$, we have

$$
h_{1}(n) \rightarrow-\left(d+d_{1} \frac{n^{2}}{l^{2}}\right)^{2}+\left(\mu I^{*}\right)^{2}+\sqrt{\triangle_{1}},
$$

where $\triangle_{1}=\left[\left(d+d_{1} \frac{n^{2}}{l^{2}}\right)^{2}-\left(\mu I^{*}\right)^{2}\right]^{2}+4\left(\mu I^{*} d\right)^{2}$. 
To prove that $\omega_{0}^{0}$ is the minimum value of $\omega_{n}^{0}$, we need to know the monotonicity of $h_{1}(n)$. So we suppose that $h_{1}(n)$ is a continuous functions of $n$, and then we can get the monotonicity of $h_{1}(n)$ by the sign of the derivative.

$$
\begin{aligned}
& h_{1}^{\prime}(n) \rightarrow-2\left(d+d_{1} \frac{n^{2}}{l^{2}}\right) d_{1} \frac{2 n}{l^{2}}+\frac{1}{\sqrt{\Delta}} 2\left[\left(d+d_{1} \frac{n^{2}}{l^{2}}\right)^{2}-\left(\mu I^{*}\right)^{2}\right]\left(d+d_{1} \frac{n^{2}}{l^{2}}\right) d_{1} \frac{2 n}{l^{2}} \\
& =2\left(d+d_{1} \frac{n^{2}}{l^{2}}\right) d_{1} \frac{2 n}{l^{2}}\left[\frac{\left(d+d_{1} \frac{n^{2}}{l^{2}}\right)^{2}-\left(\mu I^{*}\right)^{2}-\sqrt{\Delta_{1}}}{\sqrt{\Delta_{1}}}\right] .
\end{aligned}
$$

Noticing that $\left(d+d_{1} \frac{n^{2}}{l^{2}}\right)^{2}-\left(\mu I^{*}\right)^{2}-\sqrt{\Delta_{1}}<0$, we obtain $h_{1}^{\prime}(n)<0$ when $n>0$, which means that $h_{1}(n)$ is monotonically decreasing.

Now we consider the numerator of $\omega_{n}^{0}$. When $d_{2} \rightarrow 0$,

$$
\begin{aligned}
& A_{n} C z_{n}^{2}+\left(B_{n}-z_{n}^{2}\right) D_{n} \\
& =\left(d+d_{1} \frac{n^{2}}{l^{2}}+d_{2} \frac{n^{2}}{l^{2}}\right) \mu I^{*} z_{n}^{2}+\left[d_{2} \frac{n^{2}}{l^{2}}\left(d+d_{1} \frac{n^{2}}{l^{2}}\right)-z_{n}^{2}\right]\left(\mu I^{*} d_{2} \frac{n^{2}}{l^{2}}+\mu I^{*} d\right) \\
& \rightarrow\left(d+d_{1} \frac{n^{2}}{l^{2}}\right) \mu I^{*} z_{n}^{2}+\left(-z_{n}^{2}\right)\left(\mu I^{*} d\right)=d_{1} \frac{n^{2}}{l^{2}} \mu I^{*} z_{n}^{2} \geq 0, \\
& D_{n}^{2}+\left(C z_{n}\right)^{2} \rightarrow\left(\mu I^{*} d\right)^{2}+\left(\mu I^{*}\right)^{2} z_{n}^{2}>0,
\end{aligned}
$$

thus

$$
-\frac{A_{n} C z_{n}^{2}+\left(B_{n}-z_{n}^{2}\right) D_{n}}{D_{n}^{2}+\left(C z_{n}\right)^{2}} \leq 0,
$$

and the equality holds for $n=0$. Since arccosine function is a monotonically decreasing function, $\arccos \left(C_{n}\left(z_{n}\right)\right)$ takes the minimum value when $n=0$. The proof is complete.

Theorem 4. Suppose $d_{2} \rightarrow+\infty$, then for $n \in\left\{0,1, \ldots, n_{1}\right\}, \omega_{n}^{0}$ takes the minimum value when $n=0$, that is, $\omega^{*}=\omega_{0}^{0}$.

Proof. When $d_{2} \rightarrow+\infty$,

$$
\begin{aligned}
& z_{n}=\sqrt{\frac{-\left(A_{n}^{2}-2 B_{n}-C^{2}\right)+\sqrt{\triangle}}{2}}=\sqrt{\frac{1}{2} \frac{-4\left(B_{n}^{2}-D_{n}^{2}\right)}{\left(A_{n}^{2}-2 B_{n}-C^{2}\right)+\sqrt{\triangle}}} \\
& \rightarrow \sqrt{\left(\mu I^{*}\right)^{2}-\left(d+d_{1} \frac{n^{2}}{l^{2}}\right)^{2}},
\end{aligned}
$$

and

$$
C_{n}\left(z_{n}\right)=-\frac{A_{n} C z_{n}^{2}+\left(B_{n}-z_{n}^{2}\right) D_{n}}{D_{n}^{2}+\left(C z_{n}\right)^{2}} \rightarrow-\frac{d+d_{1} \frac{n^{2}}{l^{2}}}{\mu I^{*}} .
$$


To determine the monotonicity of $\omega_{n}^{0}$, we need to know the monotonicity of $\arccos C_{n}\left(z_{n}\right)$. So we suppose that $\arccos C_{n}\left(z_{n}\right)$ is a continuous functions of $n$, and then we can get the monotonicity of $\arccos C_{n}\left(z_{n}\right)$ by the sign of the derivative.

$$
\frac{d \arccos C_{n}\left(z_{n}\right)}{d n} \rightarrow \frac{1}{\sqrt{\left(1-C_{n}^{2}\right)}} \frac{d_{1} \frac{2 n}{l^{2}}}{\mu I^{*}}>0 .
$$

Obviously, when $n>0,\left(\mu I^{*}\right)^{2}-\left(d+d_{1} \frac{n^{2}}{l^{2}}\right)^{2}$ is monotonically decreasing, and $\arccos C_{n}\left(z_{n}\right)$ is monotonically increasing, thus $\omega_{n}^{0}=\frac{1}{z_{n}}\left[\arccos C_{n}\left(z_{n}\right)\right]$ is monotonically increasing, and

$$
\omega_{0}^{0}<\omega_{1}^{0}<\omega_{2}^{0}<\ldots<\omega_{n_{1}}^{0} .
$$

The proof is complete.

In fact, when the diffusive rates tend to zero, the reaction-diffusion system behaves more like an ordinary differential equation. Moreover, if the diffusive rates are sufficiently large, then all individuals move very frequently and lead the spatial distribution to its thermodynamic limit (uniform distribution) very fast. Thus, Theorems 3 and 4 provide mathematically interpretation of the two intuitionistic results. When the value of $d_{2}$ is chosen to be an appropriate size, we have the following conclusion.

We make the following assumptions

$\left(H_{1}\right)$ there exists $m, 1 \leq m \leq n_{1}$, such that $\omega_{n}^{0}$ is monotonically decreasing in $n$ for $0 \leq n \leq m$.

$\left(H_{2}\right) \omega_{n}^{0}$ is monotonically increasing in $n$ for $n>m$ with $m<1$.

Theorem 5. Suppose $\left(H_{1}\right)$ holds, then $\omega^{*}=\omega_{n}^{0}(n \neq 0)$.

Proof. Since $\omega_{n}^{0}$ is monotonically decreasing in $n$ for $0 \leq n \leq m$, it is obvious that $\omega_{0}^{0}>\omega_{n}^{0}$ for these $n$.

Suppose $\left(H_{2}\right)$ holds, then $\omega_{1}^{0}<\omega_{2}^{0}<\ldots<\omega_{n_{1}}^{0}$. We only need to compare the value of $\omega_{0}^{0}$ and $\omega_{1}^{0}$ to determine which one is $\omega^{*}$. That is, in this case, if $\omega_{1}^{0}<\omega_{0}^{0}, \omega^{*}=\omega_{1}^{0}$. If $\omega_{1}^{0}>\omega_{0}^{0}$, $\omega^{*}=\omega_{0}^{0}$.

Remark 2. Theorem 3 and 4 indicate that the first Hopf bifurcation value occurs at $n=0$ with eigenfunction 1 when the diffusion rate $d_{2}$ is sufficiently small or large enough. According to the general Hopf bifurcation theory in [487, spatial homogeneity appears. From Theorem 5, we know that if $\left(H_{1}\right)$ holds, $\omega^{*}=\omega_{n}^{0}$ with some $n \neq 0$. Thus spatially inhomogeneous oscillations are possible. In fact, we find some stable, spatially inhomogeneous oscillations near the first Hopf bifurcation of system (2) in Section 5 . 
Here, we present some discussions about the first Hopf bifurcation point. In this paper, we investigate a system consisting of three equations. However, we actually have $y(t)$ tending to a constant, thus, the system behaves like a system with only two equations about $S(t)$ and $I(t)$, whose characteristic equation is like $(12)$. So we can conclude that stable spatially inhomogeneous Hopf bifurcating solutions may exist in a system with two equations. Generally, such a system has the linearized system as the following form

$$
\frac{\partial U(x, t)}{\partial t}=D \triangle U(x, t)+A U(x, t)+B U(x, t-\tau)
$$

with $D=\left(\begin{array}{cc}d_{1} & 0 \\ 0 & d_{2}\end{array}\right), A=\left(\begin{array}{cc}a_{11} & a_{12} \\ 0 & 0\end{array}\right), B=\left(\begin{array}{cc}-b_{1} & 0 \\ b_{1} & 0\end{array}\right)$, where $a_{11}<0, a_{12}<0, b_{1}>0$.

However, for many systems, there are no spatially inhomogeneous Hopf bifurcating solutions near the first Hopf bifurcation point. For example, for the predator-prey system in [53], the Hopf bifurcation values are monotonically increasing in $n$. Therefore, the first Hopf bifurcation occurs when $n=0$, and there are no stable spatially inhomogeneous bifurcating periodic solutions. To be more specific, we study a system of the following form as studied in 53 ,

$$
\frac{\partial U(x, t)}{\partial t}=D \triangle U(x, t)+f(U(x, t), U(x, t-\tau))
$$

whose linearized system is

$$
\frac{\partial U(x, t)}{\partial t}=D \triangle U(x, t)+A U(x, t)+B U(x, t-\tau)
$$

with $D=\left(\begin{array}{cc}d_{1} & 0 \\ 0 & d_{2}\end{array}\right), A=\left(\begin{array}{cc}b_{11} & b_{12} \\ 0 & b_{22}\end{array}\right), B=\left(\begin{array}{cc}0 & 0 \\ \beta_{1} & 0\end{array}\right)$, where $b_{11}<0, b_{12}<0, b_{22} \leq$ $0, \beta_{1}>0$. We can prove that If $\left(A_{1}\right) b_{11} b_{22}+\beta_{1} b_{12}<0$ holds, the first Hopf bifurcation point is $\tau^{*}=\tau_{0}^{0}$. The derivations are similar to Theorems 3 and 4 , thus we omit them here.

Remark 3. Now we know that for some reaction-diffusion systems, there are spatially inhomogeneous Hopf bifurcating periodic solutions near the first critical value (e.g system (2)). However, for systems of the form (40), there are no spatially inhomogeneous Hopf bifurcating periodic solutions near the first critical value. Unfortunately, a clear threshold condition which determines whether or not there are spatially oscillations and the biological mechanism of the appeared stable, spatially inhomogeneous oscillations in Section 5 are both unclear yet. 

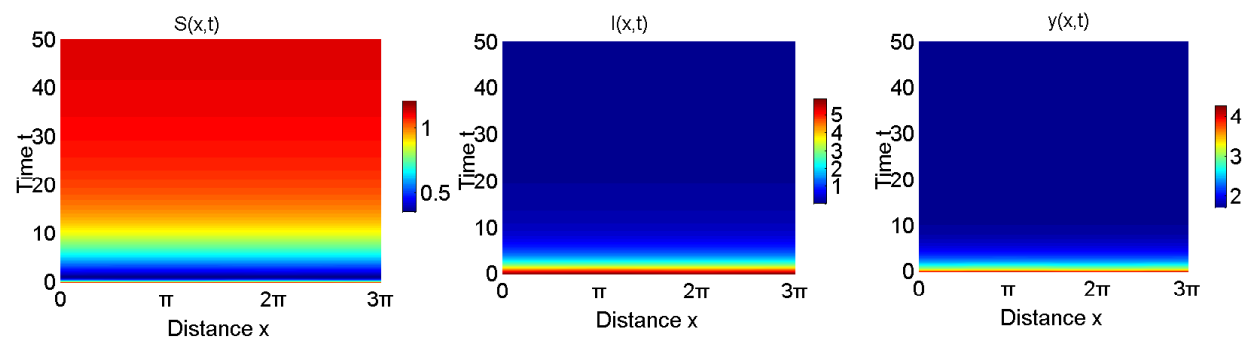

FIG. 1. When $R_{0}<1$, the disease-free equilibrium $E_{1}$ of system (2) is locally asymptotically stable.

\section{NUMERICAL SIMULATIONS}

In this section, some numerical results of system (2) are presented to verify the theoretical analysis in previous sections. In all simulations, we fix $\Omega=(0,3 \pi)$ (i.e. $l=3)$.

\subsection{Threshold dynamics by $R_{0}$}

If we choose

$$
\begin{aligned}
& \alpha=0.85, d=0.5, \mu=0.5, \gamma=0.1, \beta=0.3, \tau=1, \omega=0, \\
& d_{1}=0.05, d_{2}=0.2, d_{3}=0.06
\end{aligned}
$$

then $R_{0}=0.9579<1$, and according to the results in section 22.1 , we know that the disease-free equilibrium $E_{1}(1.1495,0,1.7185)$ is asymptotically stable (see Fig. 1).

If we choose

$$
\begin{aligned}
& \alpha=2.1, d=0.5, \mu=0.5, \gamma=0.1, \beta=0.3, \tau=1, \omega=0, \\
& d_{1}=0.05, d_{2}=0.2, d_{3}=0.06,
\end{aligned}
$$

i.e., larger $\alpha$ yields $R_{0}=5.8470>1$. Thus a unique positive equilibrium $E_{2}(1.2,5.8164,4.2457)$ appears (see Fig. 2). According to Theorem 1, the system is locally asymptotically stable.

\subsection{Spatially homogeneous oscillations induced by Hopf bifurcation}

In this section, we discuss how the stability of $E_{2}$ changes with $\omega$ varying. From Theorem 3, we know that when $d_{2}$ is sufficiently small, $\omega^{*}=\omega_{0}^{0}$, which means that spatially homogeneous Hopf bifurcation will appear near a neighborhood of $\omega^{*}$. Now we verify this 

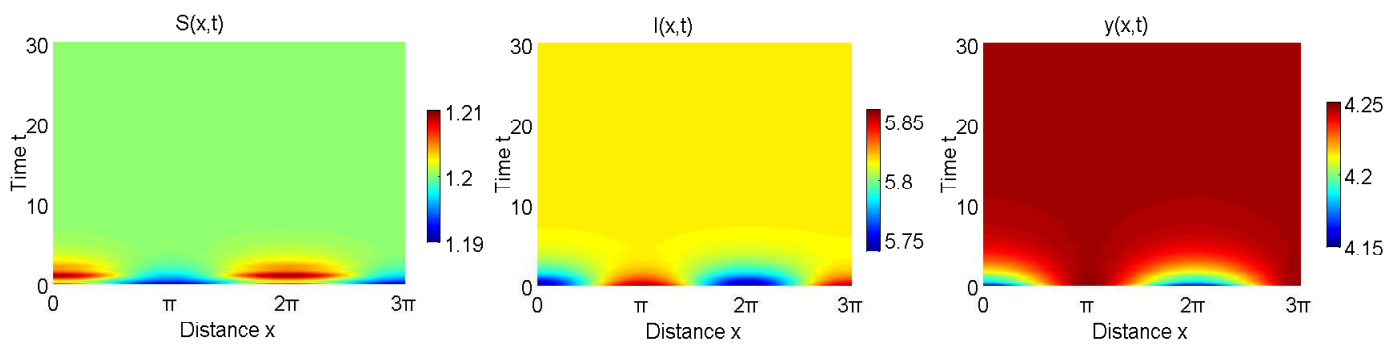

FIG. 2. When the parameters are chosen as in (43), $R_{0}>1$, and the positive equilibrium $E_{2}$ of system (2) is locally asymptotically stable.
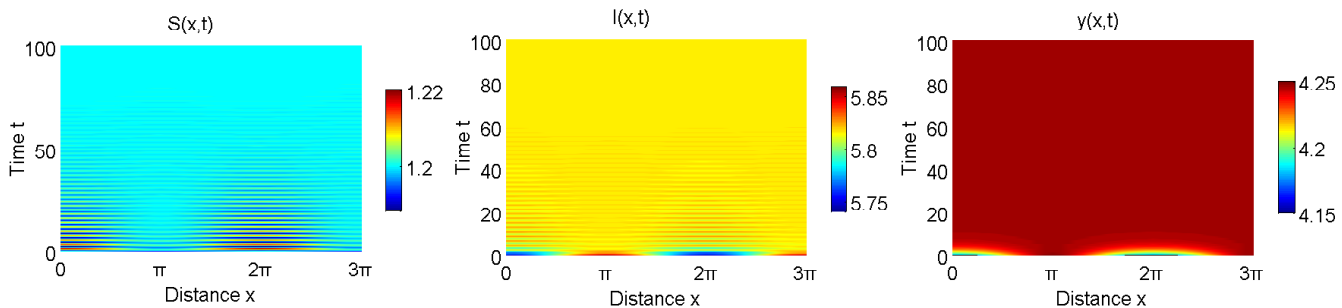

FIG. 3. When $d_{2}=0.2$ and $\omega=0.52<\omega^{*}=0.5401$, the positive equilibrium $E_{2}$ of system (2) is locally asymptotically stable.

numerically with a small value of $d_{2}$. We fix the parameters

$$
\begin{aligned}
& \alpha=2.1, d=0.5, \mu=0.5, \gamma=0.1, \beta=0.3, \tau=1, \\
& d_{1}=0.05, d_{2}=0.2, d_{3}=0.06,
\end{aligned}
$$

and vary the freely-moving delay $\omega$. From calculation, we find that when $n \leq 21$, Eq. (16) has one positive root, i.e., Hopf bifurcations induced by delay occur for $n \leq 21$. By calculation, when $n_{0}=0, \omega_{0}^{0}=\min _{n \in\{0,1, \ldots, 21\}}\left\{\omega_{n}^{0}\right\}$, that is, $\omega^{*}=\omega_{0}^{0}=0.5401$ is the first Hopf bifurcation point of the freely-moving delay. Hence from Theorem 1, we know that when $\omega<\omega^{*}, E_{2}$ is asymptotically stable, which is shown in Fig. 3. When $\omega$ passes through the critical value $\omega^{*}, E_{2}$ loses its stability and system (2) undergoes a spatially homogeneous Hopf bifurcation near the positive equilibrium $E_{2}$. In addition, it follows from (36) that $c_{1}(0)=-0.00046-0.00623 i$. From Theorem 2, we know that the spatially homogeneous Hopf bifurcation is supercritical, the bifurcating periodic solutions are stable (see Fig. 4). In these figures, ignoring the transient states, we only demonstrate the part of stable periodic oscillations.

From Theorem 4, we know that when $d_{2}$ is large enough, $\omega^{*}=\omega_{0}^{0}$, and spatially homo- 

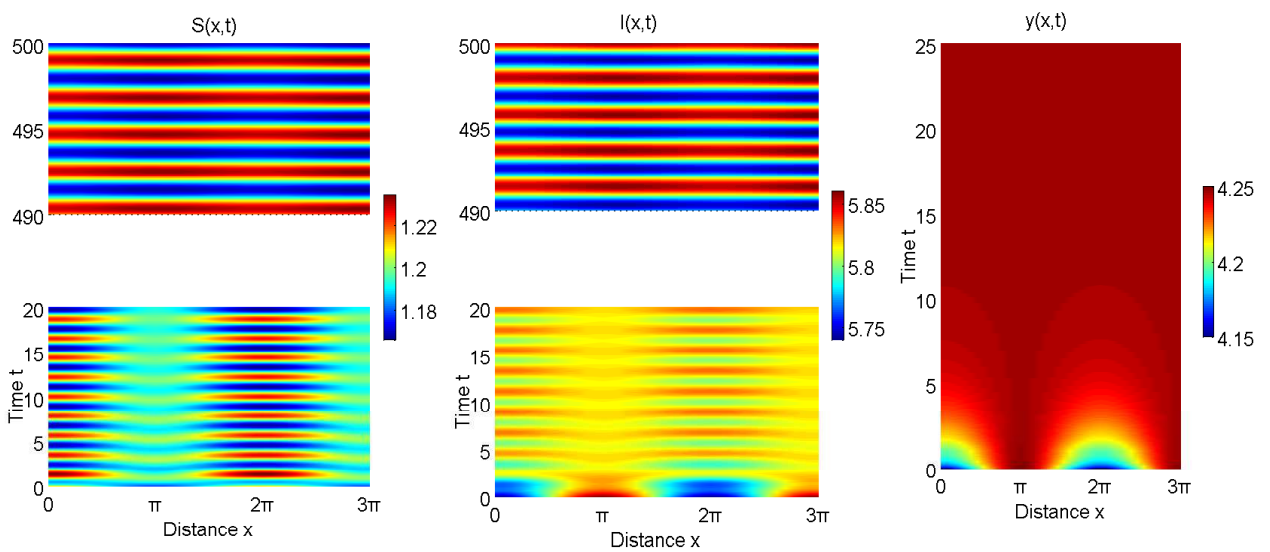

FIG. 4. When $d_{2}=0.2$, and $\omega=0.541>\omega^{*}=\omega_{0}^{0}=0.5401$, the positive equilibrium $E_{2}$ of (2) loses its stability and the spatially homogeneous bifurcating periodic solutions are stable.
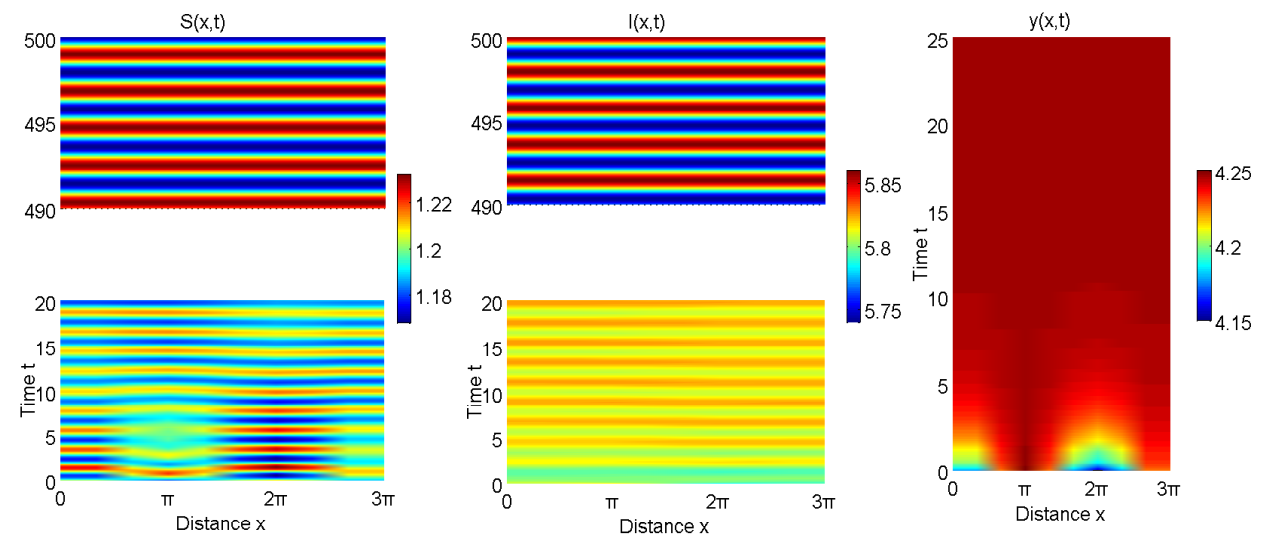

FIG. 5. When $d_{2}=40$, and $\omega=0.541>\omega^{*}=\omega_{3}^{0}=0.5401$, the spatially homogeneous bifurcating periodic solutions are stable.

geneous Hopf bifurcation will appear. Now we verify the result numerically. Let

$$
\begin{aligned}
& \alpha=2.1, d=0.5, \mu=0.5, \gamma=0.1, \beta=0.3, \tau=1, \\
& d_{1}=0.05, d_{2}=40, d_{3}=0.06
\end{aligned}
$$

Similar to the aforementioned calculation, we find that when $n \leq 20$, Eq. (16) has one positive root, i.e., Hopf bifurcations induced by delay occur for $n \leq 20$. Moreover, we have $\omega^{*}=\omega_{0}^{0}=0.5401$, and $c_{1}(0)=-0.00046-0.00623 i$. From Theorem 2 , we know that the spatially homogeneous Hopf bifurcation is supercritical, the bifurcating periodic solutions are stable (see Fig. 5). 
a)

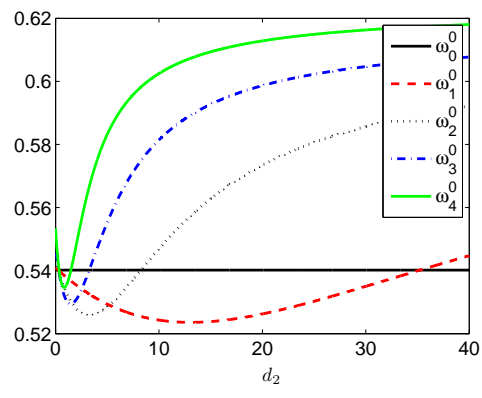

b)

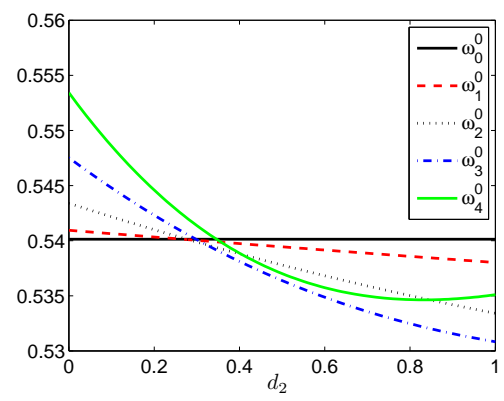

FIG. 6. The curves of $\omega_{n_{0}}^{0}, 0 \leq n_{0} \leq 4$, are drawn when $d_{2}$ varies.

\subsection{Spatially inhomogeneous oscillations induced by Hopf bifurcation}

When $d_{2}$ increases passing through some proper values, $\omega^{*}$ may not always be $\omega_{0}^{0}$, and thus the spatial structure may change. In the following, we fix the parameters

$$
\alpha=2.1, d=0.5, \mu=0.5, \gamma=0.1, \beta=0.3, \tau=1, d_{1}=0.05, d_{3}=0.06
$$

and vary $d_{2}$. Now we demonstrate the effect of the diffusion coefficient $d_{2}$ on the critical value $\omega^{*}$, and thus we can observe how the spatial structure changes with varying $d_{2}$.

In fact, by calculation, we can verify that when $d_{2} \leq 0.29, \omega_{n}^{0}$ is monotonically increasing in $n$ for $n \leq n_{1}$, and thus $\omega^{*}=\omega_{0}^{0}$. When $0.29<d_{2}<15,\left(H_{1}\right)$ holds, and thus we can conclude that $\omega^{*}=\omega_{n}^{0}$ for some $n \neq 0$. If $d_{2}>15,\left(H_{2}\right)$ holds. Comparing the value of $\omega_{1}^{0}$ with $\omega_{0}^{0}$, we have $\omega_{1}^{0}<\omega_{0}^{0}$ when $15 \leq d_{2} \leq 35$, and $\omega_{1}^{0}>\omega_{0}^{0}$ when $d_{2}>35$. Fig. 6 illustrates the function of $\omega_{n_{0}}^{0}\left(d_{2}\right)$ when $d_{2}$ varies, and thus we can see which one is the first bifurcation value easily when $d_{2}$ are set to be certain values. We can see that when $d_{2}$ is small, $\omega^{*}=\omega_{0}^{0}$. With diffusion coefficient $d_{2}$ increasing, $\omega^{*}$ can be the value of $\omega_{3}^{0}, \omega_{2}^{0}$, or $\omega_{1}^{0}$. Keep increasing $d_{2}$ to make it large enough, $\omega^{*}=\omega_{0}^{0}$.

We choose certain values for $d_{2}\left(d_{2}=0.4, d_{2}=2.5, d_{2}=5.5\right)$, and give corresponding simulation results (see Fig. 7, Fig. 8 and Fig. 9 respectively). For the convenience of our statement, we record the data of these cases in Table 1, such as the bifurcation points and $C_{1}(0)$ determining the properties of bifurcations.

For the numerical simulations in Fig. 4, 5, 7, 8, and 9, the initial conditions are all $S(x, t)=$ $1.2+0.01 \cos x, I(x, t)=5.8-0.06 \cos x, y(x, t)=4.2-0.05 \cos x$, and the parameters

are all the same as in (46) except $d_{2}$ and $\omega$. However, we can see that the dynamical behaviors are totally different from each other. When $d_{2}=0.2, \omega^{*}=\omega_{0}^{0}=0.5401$, a spatially 
TABLE I. Hopf bifurcations for some values of $d_{2}$ when $l=3$.

\begin{tabular}{lllll}
\hline$d_{2}$ & First bifurcation point $\omega^{*}$ & $c_{1}(0)$ & Stability of periodic solutions Illustrated in \\
\hline 0.2 & $\omega_{0}^{0}=0.5401$ & $-0.00046-0.00623 \mathrm{i}$ & Stable & Fig. 4 \\
0.4 & $\omega_{3}^{0}=0.5381$ & $-0.00034-0.00024 \mathrm{i}$ & Stable & Fig. 7 \\
2.5 & $\omega_{2}^{0}=0.5265$ & $-0.00029+0.00001 \mathrm{i}$ & Stable \\
5.5 & $\omega_{1}^{0}=0.5286$ & $-0.00032-0.00015 \mathrm{i}$ & Stable & Fig. 8 \\
40 & $\omega_{0}^{0}=0.5401$ & $-0.00046-0.00623 \mathrm{i}$ & Stable & Fig. 9 \\
\hline
\end{tabular}
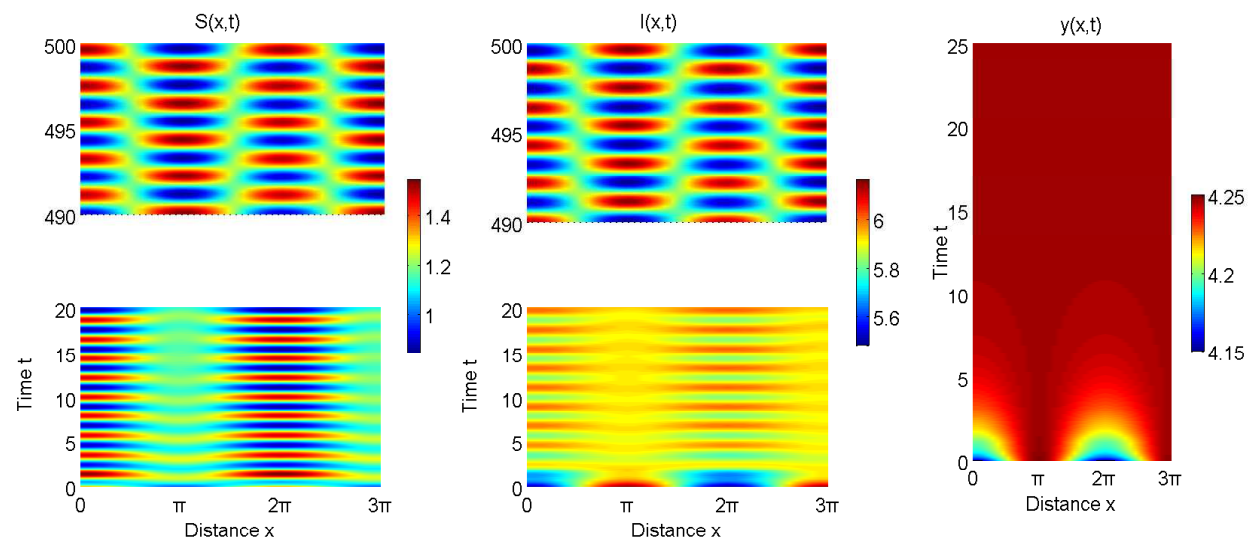

FIG. 7. When $d_{2}=0.4$, and $\omega=0.54>\omega^{*}=\omega_{3}^{0}=0.5381$, the spatially inhomogeneous bifurcating periodic solutions of shape as the corresponding eigenfunction $\cos (x)$ are stable.
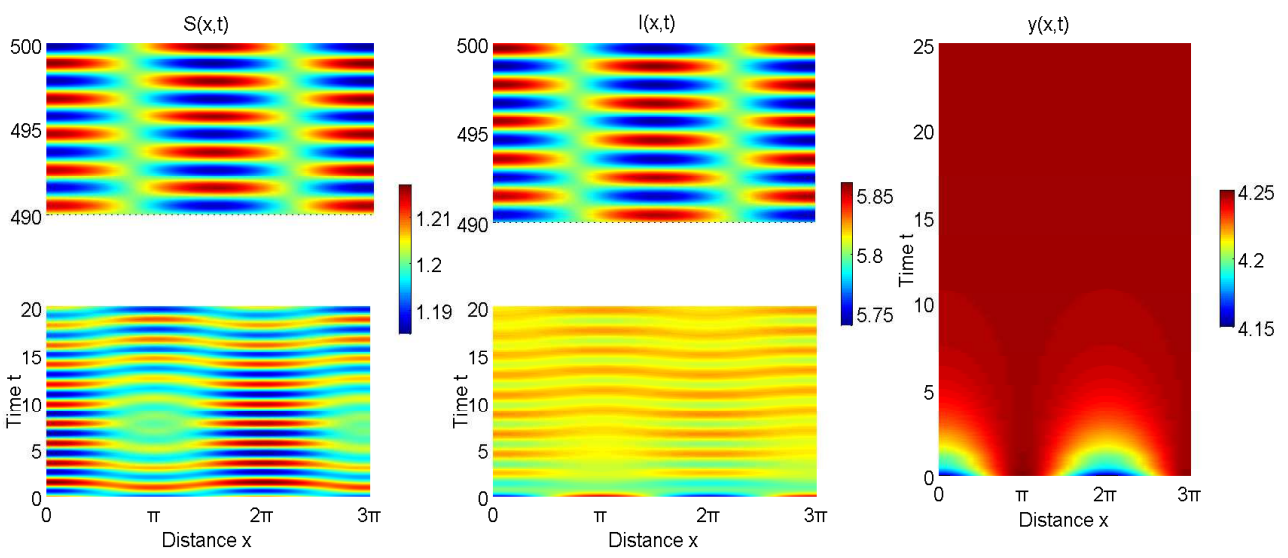

FIG. 8. When $d_{2}=2.5$, and $\omega=0.53>\omega^{*}=\omega_{2}^{0}=0.5265$, the spatially inhomogeneous bifurcating periodic solutions of shape as $\cos \left(\frac{2}{3} x\right)$ are stable. 

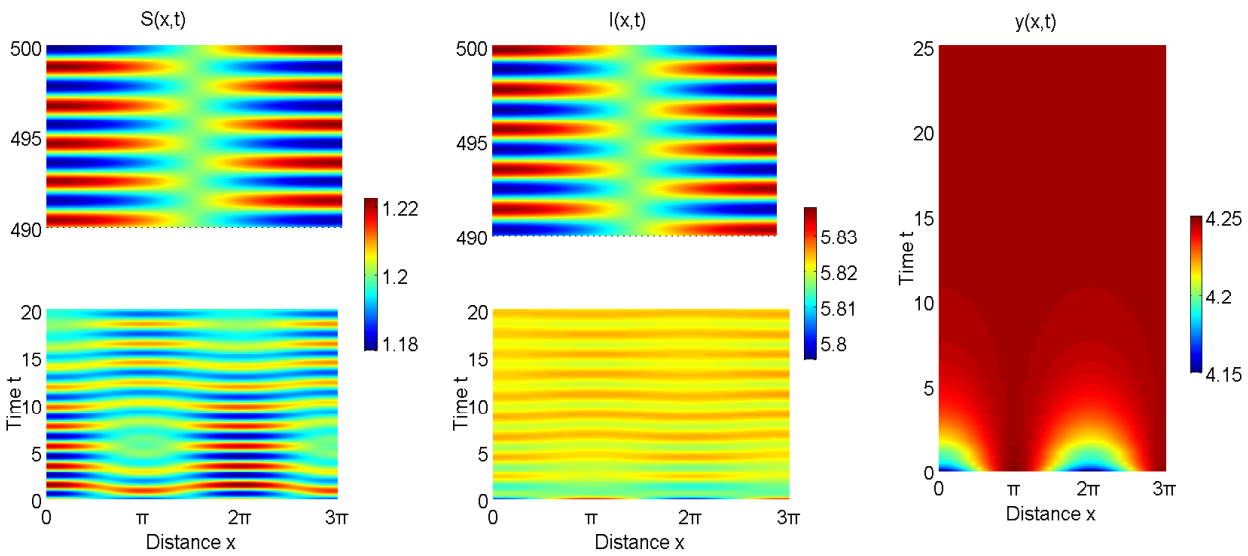

FIG. 9. When $d_{2}=5.5$, and $\omega=0.53>\omega^{*}=\omega_{1}^{0}=0.5286$, the spatially inhomogeneous bifurcating periodic solutions of shape as $\cos \left(\frac{1}{3} x\right)$ are stable.

TABLE II. Hopf bifurcations for some values of $d_{2}$ when $l=2$.

\begin{tabular}{lcccc}
\hline$d_{2}$ & First bifurcation point $\omega^{*}$ & $c_{1}(0)$ & Stability of periodic solutions & spatial profile \\
\hline 0.2 & $\omega_{0}^{0}=0.5401$ & $-0.00046-0.00623 \mathrm{i}$ & Stable & homogeneous \\
0.4 & $\omega_{2}^{0}=0.5381$ & $-0.00051-0.00036 \mathrm{i}$ & Stable & inhomogeneous \\
5.5 & $\omega_{1}^{0}=0.5245$ & $-0.00039+0.00093 \mathrm{i}$ & Stable & inhomogeneous \\
20 & $\omega_{0}^{0}=0.5401$ & $-0.00046-0.00623 \mathrm{i}$ & Stable & homogeneous \\
\hline
\end{tabular}

homogeneous periodic solution occurs near the positive equilibrium $E_{2}$. When $d_{2}=0.4$, $\omega^{*}=\omega_{3}^{0}=0.5381$, a spatially inhomogeneous periodic solution with spatial profile $\cos (x)$ occurs. When $d_{2}=2.5$, and $\omega^{*}=\omega_{2}^{0}=0.5265$, the spatially inhomogeneous periodic solution is of the shape $\cos \left(\frac{2}{3} x\right)$. When $d_{2}=5.5, \omega^{*}=\omega_{1}^{0}=0.5286$, the spatially inhomogeneous periodic solution is of the shape $\cos \left(\frac{1}{3} x\right)$. When $d_{2}=40, \omega^{*}=\omega_{0}^{0}=0.5401$, a spatially homogeneous periodic solution occurs. Thus, we can tell that the diffusion coefficient $d_{2}$ have direct effect on the dynamics of system (2).

If we fix $\Omega=(0,2 \pi)$ (i.e. $l=2)$, we can also get some similar simulating results. We ignore the presentation of the corresponding figures, and just record the data of these results in Table 2. 


\section{CONCLUSION REMARKS}

Spatio-temporal distribution of species is a key problem in the population dynamics. In this paper, we find the diffusion-driven spatial inhomogeneity and temporal oscillations near a Hopf bifurcation, which are induced by the freely-moving delay in an stage-structured epidemic model. Particularly, the following issues are studied from the view of bifurcation analysis.

We give the threshold dynamics characterizing by basic reproduction ratio $R_{0}$ : when $R_{0}<1$, the disease-free constant equilibrium $E_{1}$ is locally asymptotically stable; and when $R_{0}>1$, in the absence of freely-moving delay, the endemic equilibrium is locally stable.

Using the freely-moving delay as the bifurcation parameter, we show that the delay can destabilize the positive constant equilibrium, and induces Hopf bifurcations. In fact, the only possible bifurcation near this equilibrium is Hopf bifurcation, that is, we exclude the existence of Turing bifurcation, which usually induces spatial inhomogeneity.

By using the normal form theory and the center manifold Theorem, we derive formulae to determine the properties of spatially bifurcating periodic solutions.

When the freely-moving delay passes through the critical value $\omega^{*}=\omega_{n_{0}}^{0}$ for some $n_{0}$, the positive constant equilibrium loses its stability and homogeneous or inhomogeneous Hopf bifurcations occur. If $n_{0}=0$, system (2) occurs spatially homogenous Hopf bifurcating solution. For $n_{0} \neq 0$, system (2) exhibits spatially inhomogeneous Hopf bifurcating solution. We list a sequence of results in Table 1 and illustrate the curves in Fig. 6, where we show the relation between $\omega^{*}$ and the diffusion coefficient, when all the other parameters are fixed. Theoretically, we have proved in Theorem 3 that the first bifurcating oscillation is always spatially homogeneous when this coefficient is sufficiently small.

With diffusion coefficient $d_{2}$ increasing, from Theorem 5, if $\left(H_{1}\right)$ holds, $\omega^{*}=\omega_{n}^{0}(n \neq 0)$. Numerical simulation shows that $\omega^{*}$ can be the value of $\omega_{3}^{0}, \omega_{2}^{0}$, or $\omega_{1}^{0}$, and different kinds of spatially inhomogeneous oscillations with different shapes come out. From Theorem 4 , keep increasing $d_{2}$ to make it large enough, then $\omega^{*}=\omega_{0}^{0}$, and the spatial oscillations is homogeneous again. That is, in the process of increasing the diffusion coefficient $d_{2}$, the spatial structure switches from homogeneous to inhomogeneous, and then back to homogeneous, which is in accordance with the biological meaning: very large speed of random diffusion will eliminate the spatially inhomogeneous distribution of a species. 
As shown clearly in Fig. 6 a), every two Hopf bifurcation curves intersect at a double Hopf bifurcation point. This is a very interesting problem, because double Hopf bifurcation usually leads the system to quasi-periodical oscillations with two or three frequencies, i.e., oscillating on two or three dimensional torus. Moreover, double Hopf bifurcation may induce chaos to a system [54]. This is left as a further study.

In a previous work [21], we have investigated an SEIR model with stage structure and freely-moving delay from the point of view of bifurcation analysis. We showed that increasing the delay could destabilize the endemic equilibrium, and induce Hopf bifurcations and stable temporal periodic solutions. Now, we incorporate diffusion terms into such a system and find that they may induce not only temporal oscillations but also spatial oscillations. From (11), we can find that varying the diffusion rate of mature stage $d_{3}$ will not change any local bifurcation results. By fixing the diffusion rate of the susceptible $d_{1}$, we vary the diffusion rate of the infected $d_{2}$. From Theorem 3 and 4 , when $d_{2}$ is sufficiently small or sufficiently large, there are only spatially homogeneous oscillations. In such a situation, our diffusive model behaves exactly the same as the DDE system in [21] does. However, when $d_{2}$ is chosen as an appropriate size, there are spatially inhomogenous oscillations. Hence the population distribution is totally changed, which cannot be described by the DDE system in [21].

\section{ACKNOWLEDGEMENTS}

The author deeply appreciates the time and effort that the editor and referees spend on reviewing the manuscript. This research is supported by National Natural Science Foundation of China (11371112, 11701120).

\section{Appendix A: Computation of the coefficients $\mu_{2}, \beta_{2}, T_{2}$}

Throughout the section, we compute the coefficients $\mu_{2}, \beta_{2}, T_{2}$ to determine the properties of Hopf bifurcation.

From section 22.2, we know that $\Lambda_{n_{0}}= \pm i z_{n_{0}} \omega_{n_{0}}= \pm i z^{*} \omega^{*}$ are eigenvalues of $A\left(\omega^{*}\right)$ and thus they are also eigenvalues of $A^{*}$. We first need to compute the eigenvector of $A(0)$ and $A^{*}$ corresponding to $i z^{*} \omega^{*}$ and $-i z^{*} \omega^{*}$, respectively. Let $P$ and $P^{*}$ be the center subspace, namely the generalized eigenspace of $A\left(\omega^{*}\right)$ and $A^{*}$ associated with $\Lambda_{n_{0}}$, respectively. 
Moreover, $P^{*}$ is the adjoint space of $P$ and $\operatorname{dim} P=\operatorname{dim} P^{*}=2$.

By direct computations, we get the following results.

Lemma 4. Let

$$
\begin{aligned}
& \xi_{1}=\frac{\mu I^{*} e^{-i z^{*} \omega^{*}}}{d_{2} \frac{n_{0}^{2}}{l^{2}}+i z^{*}}, \quad \xi_{2}=0, \\
& \eta_{1}=-\frac{-\mu S^{*}+\gamma}{-d_{2} \frac{n_{0}^{2}}{l^{2}}+i z^{*}}, \eta_{2}=-\frac{\alpha-\alpha e^{-d \tau} e^{i z^{*} \omega^{*} \tau^{*}}}{-2 \beta y^{*}-d_{3} \frac{n_{0}^{2}}{l^{2}}+\alpha e^{-d \tau} e^{i z^{*} \omega^{*} \tau^{*}}+i z^{*}},
\end{aligned}
$$

then

$$
p_{1}(\theta)=\left(1, \xi_{1}, \xi_{2}\right)^{T} e^{i z^{*} \omega^{*} \theta}, \quad p_{2}(\theta)=\overline{p_{1}(\theta)}, \quad \theta \in\left[-\tau^{*}, 0\right],
$$

is a basis of $P$ with $\Lambda_{n}$ and

$$
q_{1}(s)=\left(1, \eta_{1}, \eta_{2}\right) e^{-i z^{*} \omega^{*} s}, \quad q_{2}(s)=\overline{q_{1}(s)}, \quad s \in\left[0, \tau^{*}\right],
$$

is a basis of $P^{*}$ with $\Lambda_{n}$.

Let $\Phi=\left(\Phi_{1}, \Phi_{2}\right)$ is obtained by separating the real and imaginary parts of $p_{1}(\theta)$, and $\Phi$ is also the basis of $P$. Similarly, $\Phi^{*}=\left(\Phi_{1}^{*}, \Phi_{2}^{*}\right)^{T}$ is also the basis of $P^{*}$. Then, direct calculations yield that

$$
\begin{gathered}
\Phi_{1}(\theta)=\frac{p_{1}(\theta)+p_{2}(\theta)}{2}=\operatorname{Re}\left(\begin{array}{c}
e^{i z^{*} \omega^{*} \theta} \\
\xi_{1} e^{i z^{*} \omega^{*} \theta} \\
\xi_{2} e^{i z^{*} \omega^{*} \theta}
\end{array}\right)=\left(\begin{array}{c}
\cos \left(z^{*} \omega^{*} \theta\right) \\
\frac{\mu I^{*}\left[\cos \left(z^{*} \omega^{*}(\theta-1)\right) d_{2} \frac{n_{0}^{2}}{l^{2}}+z^{*} \sin \left(z^{*} \omega^{*}(\theta-1)\right)\right]}{\left(d_{2} \frac{n_{0}^{2}}{l^{2}}\right)^{2}+z^{* 2}} \\
0
\end{array}\right) \\
\Phi_{2}(\theta)=\frac{p_{1}(\theta)-p_{2}(\theta)}{2 i}=\operatorname{Im}\left(\begin{array}{c}
e^{i z^{*} \omega^{*} \theta} \\
\xi_{1} e^{i z^{*} \omega^{*} \theta} \\
\xi_{2} e^{i z^{*} \omega^{*} \theta}
\end{array}\right)=\left(\begin{array}{c}
\sin \left(z^{*} \omega^{*} \theta\right) \\
\frac{\mu I^{*}\left[\sin \left(z^{*} \omega^{*}(\theta-1)\right) d_{2} \frac{n_{0}^{2}}{l^{2}}-z^{*} \cos \left(z^{*} \omega^{*}(\theta-1)\right)\right]}{\left(d_{2} \frac{n_{0}^{2}}{l^{2}}\right)^{2}+z^{* 2}} \\
0 \\
\Phi_{1}^{*}(s)=\frac{q_{1}(s)+q_{2}(s)}{2}=\operatorname{Re}\left(\begin{array}{c}
e^{-i z^{*} \omega^{*} s} \\
\eta_{1} e^{-i z^{*} \omega^{*} s} \\
\eta_{2} e^{-i z^{*} \omega^{*} s}
\end{array}\right) \\
\Phi_{2}^{*}(s)=\frac{q_{1}(s)+q_{2}(s)}{2 i}=\operatorname{Im}\left(\begin{array}{c}
\frac{\left(-\mu S^{*}+\gamma\right)\left[\cos \left(z^{*} \omega^{*} s\right) d_{2} \frac{n_{0}^{2}}{l^{2}}+z^{*} \sin \left(z^{*} \omega^{*} s\right)\right]}{\left(d_{2} \frac{n_{0}^{2}}{l^{2}}\right)^{2}+z^{* 2}} \\
\Phi_{13}^{*}(s) \\
\eta_{1} e^{-i z^{*} \omega^{*} s} \\
\eta_{2} e^{-i z^{*} \omega^{*} s}
\end{array}\right)
\end{array}\right)
\end{gathered}
$$


where

$\Phi_{13}^{*}(s)=-\frac{1}{M}\left(\alpha \cos \left(z^{*} \omega^{*} s\right)-\alpha e^{-d \tau} \cos \left(z^{*} \omega^{*}\left(\tau^{*}-s\right)\right)\right)\left(-2 \beta y^{*}-d_{3} n_{0}^{2} / l^{2}+a e^{-d \tau} \cos \left(z^{*} \omega^{*} \tau^{*}\right)\right)-$ $\left(a \sin \left(z^{*} \omega^{*} s\right)+\alpha e^{-d \tau} \sin \left(z^{*} \omega^{*}\left(\tau^{*}-s\right)\right)\right)\left(\alpha e^{-d \tau} \sin \left(z^{*} \omega^{*} \tau^{*}\right)+z^{*}\right)$,

$\Phi_{23}^{*}(s)=\frac{1}{M}\left(\alpha \cos \left(z^{*} \omega^{*} s\right)-\alpha e^{-d \tau} \cos \left(z^{*} \omega^{*}\left(\tau^{*}-s\right)\right)\right)\left(\alpha e^{-d \tau} \sin \left(z^{*} \omega^{*} \tau^{*}\right)+z^{*}\right)+\left(a \sin \left(z^{*} \omega^{*} s\right)+\right.$ $\left.\alpha e^{-d \tau} \sin \left(z^{*} \omega^{*}\left(\tau^{*}-s\right)\right)\right)\left(-2 \beta y^{*}-d_{3} n_{0}^{2} / l^{2}+a e^{-d \tau} \cos \left(z^{*} \omega^{*} \tau^{*}\right)\right)$, and

$$
M=\left(-2 \beta y^{*}-d_{3} \frac{n_{0}^{2}}{l^{2}}+\alpha e^{-d \tau} \cos \left(z^{*} \omega^{*} \tau^{*}\right)\right)^{2}+\left(\alpha e^{-d \tau} \sin \left(z^{*} \omega^{*} \tau^{*}\right)+z^{*}\right)^{2} .
$$

According to the bilinear form (35), we can compute

$$
\begin{aligned}
& \left(\Phi_{1}^{*}, \Phi_{1}\right)=1+\frac{\left(-\mu S^{*}+\gamma\right)\left(d_{2} \frac{n_{0}^{2}}{l^{2}}\right) \mu I^{*}\left[\cos \left(z^{*} \omega^{*}\right) d_{2} \frac{n_{0}^{2}}{l^{2}}-z^{*} \sin \left(z^{*} \omega^{*}\right)\right]}{\left(\left(d_{2} \frac{n_{0}^{2}}{l^{2}}\right)^{2}+z^{* 2}\right)^{2}} \\
& +\frac{1}{2} \omega^{*} \mu I^{*}\left[\frac{\left(-\mu S^{*}+\gamma\right)\left(\left(\frac{\sin \left(z^{*} \omega^{*}\right)}{z^{*} \omega^{*}}+\cos \left(z^{*} \omega^{*}\right)\right) d_{2} \frac{n_{0}^{2}}{l^{2}}+z^{*} \sin \left(z^{*} \omega^{*}\right)\right)}{\left(d_{2} \frac{n_{0}^{2}}{l^{2}}\right)^{2}+z^{* 2}}\right. \\
& \left.-\left(\frac{\sin \left(z^{*} \omega^{*}\right)}{z^{*} \omega^{*}}+\cos \left(z^{*} \omega^{*}\right)\right)\right] \\
& \left(\Phi_{1}^{*}, \Phi_{2}\right)=\frac{\left(-\mu S^{*}+\gamma\right)\left(d_{2} \frac{n_{0}^{2}}{l^{2}}\right) \mu I^{*}\left[-\sin \left(z^{*} \omega^{*}\right) d_{2} \frac{n_{0}^{2}}{l^{2}}-z^{*} \cos \left(z^{*} \omega^{*}\right)\right]}{\left(\left(d_{2} \frac{n_{0}^{2}}{l^{2}}\right)^{2}+z^{* 2}\right)^{2}} \\
& +\frac{1}{2} \omega^{*} \mu I^{*}\left[\frac{\left(-\mu S^{*}+\gamma\right)\left(\left(-\sin \left(z^{*} \omega^{*}\right)\right) d_{2} \frac{n_{0}^{2}}{l^{2}}-z^{*}\left(\frac{\sin \left(z^{*} \omega^{*}\right)}{z^{*} \omega^{*}}-\cos \left(z^{*} \omega^{*}\right)\right)\right)}{\left(d_{2} \frac{n_{0}^{2}}{l^{2}}\right)^{2}+z^{* 2}}+\sin \left(z^{*} \omega^{*}\right)\right], \\
& \left(\Phi_{2}^{*}, \Phi_{1}\right)=\frac{\left(-\mu S^{*}+\gamma\right) z^{*} \mu I^{*}\left[\cos \left(z^{*} \omega^{*}\right) d_{2} \frac{n_{0}^{2}}{l^{2}}-z^{*} \sin \left(z^{*} \omega^{*}\right)\right]}{\left(\left(d_{2} \frac{n_{0}^{2}}{l^{2}}\right)^{2}+z^{* 2}\right)^{2}} \\
& +\frac{1}{2} \omega^{*} \mu I^{*}\left[\frac{\left(-\mu S^{*}+\gamma\right)\left(\left(-\sin \left(z^{*} \omega^{*}\right)\right) d_{2} \frac{n_{0}^{2}}{l^{2}}+z^{*}\left(\frac{\sin \left(z^{*} \omega^{*}\right)}{z^{*} \omega^{*}}+\cos \left(z^{*} \omega^{*}\right)\right)\right)}{\left(d_{2} \frac{n_{0}^{2}}{l^{2}}\right)^{2}+z^{* 2}}+\sin \left(z^{*} \omega^{*}\right)\right], \\
& \left(\Phi_{2}^{*}, \Phi_{2}\right)=\frac{\left(-\mu S^{*}+\gamma\right) z^{*} \mu I^{*}\left[-\sin \left(z^{*} \omega^{*}\right) d_{2} \frac{n_{0}^{2}}{l^{2}}-z^{*} \cos \left(z^{*} \omega^{*}\right)\right]}{\left(\left(d_{2} \frac{n_{0}^{2}}{l^{2}}\right)^{2}+z^{* 2}\right)^{2}} \\
& +\frac{1}{2} \omega^{*} \mu I^{*}\left[\frac{\left(-\mu S^{*}+\gamma\right)\left(\left(\frac{\sin \left(z^{*} \omega^{*}\right)}{z^{*} \omega^{*}}-\cos \left(z^{*} \omega^{*}\right)\right) d_{2} \frac{n_{0}^{2}}{l^{2}}-z^{*}\left(\sin \left(z^{*} \omega^{*}\right)\right)\right)}{\left(d_{2} \frac{n_{0}^{2}}{l^{2}}\right)^{2}+z^{* 2}}\right. \\
& \left.-\left(\frac{\sin \left(z^{*} \omega^{*}\right)}{z^{*} \omega^{*}}-\cos \left(z^{*} \omega^{*}\right)\right)\right] .
\end{aligned}
$$

Now we define

$$
\left(\Phi^{*}, \Phi\right)=\left(\begin{array}{ll}
\left(\Phi_{1}^{*}, \Phi_{1}\right) & \left(\Phi_{1}^{*}, \Phi_{2}\right) \\
\left(\Phi_{2}^{*}, \Phi_{1}\right) & \left(\Phi_{2}^{*}, \Phi_{2}\right)
\end{array}\right),
$$

and construct a new basis $\Psi$ for $P^{*}$ by $\Psi=\left(\Psi_{1}, \Psi_{2}\right)^{T}=\left(\Phi^{*}, \Phi\right)^{-1} \Phi^{*}$. Then, $\Phi$ and $\Psi$ are satisfied $(\Psi, \Phi)=I_{3}$. 
Denote

$$
b_{n}=\frac{\cos \frac{n}{l} x}{\left\|\cos \frac{n}{l} x\right\|}= \begin{cases}\sqrt{\frac{1}{l \pi}}, & n=0, \\ \sqrt{\frac{2}{l \pi}} \cos \frac{n}{l} x, & n \geq 1,\end{cases}
$$

where

$$
\left\|\cos \frac{n}{l} x\right\|=\left(\int_{0}^{l \pi} \cos ^{2} \frac{n x}{l} d x\right)^{\frac{1}{2}}
$$

Let

$$
\beta_{n}^{1}=\left(\begin{array}{c}
b_{n} \\
0 \\
0
\end{array}\right), \quad \beta_{n}^{2}=\left(\begin{array}{c}
0 \\
b_{n} \\
0
\end{array}\right), \beta_{n}^{3}=\left(\begin{array}{c}
0 \\
0 \\
b_{n}
\end{array}\right),
$$

and $f_{n}=\left(\beta_{n}^{1}, \beta_{n}^{2}, \beta_{n}^{3}\right)$. Define $c \cdot f_{n}=c_{1} \beta_{n}^{1}+c_{2} \beta_{n}^{2}+c_{3} \beta_{n}^{3}$ for $c=\left(c_{1}, c_{2}, c_{3}\right)^{T} \in C\left(\left[-\tau^{*}, 0\right], X\right)$. Then the center subspace of the linear equation 32 is given by $P_{C N} \mathcal{C}$, where

$$
P_{C N} \phi=\Phi\left(\Psi,\left\langle\phi, f_{n_{0}}\right\rangle\right) \cdot f_{n_{0}}, \quad \phi \in \mathcal{C}
$$

Let $\mathcal{C}=P_{C N} \mathcal{C} \oplus P_{S} \mathcal{C}$, where $P_{S} \mathcal{C}$ denotes the complement subspace of $P_{C N} \mathcal{C}$ in $\mathcal{C}$,

$$
\langle u, v\rangle:=\frac{1}{l \pi} \int_{0}^{l \pi} u_{1} \overline{v_{1}} d x+\frac{1}{l \pi} \int_{0}^{l \pi} u_{2} \overline{v_{2}} d x+\frac{1}{l \pi} \int_{0}^{l \pi} u_{3} \overline{v_{3}} d x
$$

for $u=\left(u_{1}, u_{2}, u_{3}\right)^{T}, v=\left(v_{1}, v_{2}, v_{3}\right)^{T}, u, v \in X$ and $\left\langle\phi, f_{n}\right\rangle=\left(\left\langle\phi, \beta_{n}^{1}\right\rangle,\left\langle\phi, \beta_{n}^{2}\right\rangle,\left\langle\phi, \beta_{n}^{3}\right\rangle\right)^{T}$.

Let $A\left(\omega^{*}\right)$ be the infinitesimal generator induced by the solution of (32). Then (28) can be rewritten as

$$
\frac{d U(t)}{d t}=A\left(\omega^{*}\right) U_{t}+X_{0} F\left(U_{t}, \nu\right)
$$

where

$$
X_{0}(\theta)=\left\{\begin{array}{l}
0, \theta \in\left[-\tau^{*}, 0\right) \\
I, \theta=0
\end{array}\right.
$$

Using the decomposition $\mathcal{C}=P_{C N} \mathcal{C} \oplus P_{S} \mathcal{C}$ and $(\mathrm{A} 2)$, the solution of (31) can be written as

$$
U_{t}=\Phi\left(\begin{array}{c}
x_{1}(t) \\
x_{2}(t)
\end{array}\right) \cdot f_{n_{0}}+h\left(x_{1}, x_{2}, \nu\right)
$$

where $\left(x_{1}(t), x_{2}(t)\right)^{T}=\left(\Psi,\left\langle U_{t}, f_{n_{0}}\right\rangle\right), h\left(x_{1}, x_{2}, \nu\right) \in P_{S} \mathcal{C}$, and $h(0,0,0)=D h(0,0,0)=0$. In fact, the solution of (31) on the center manifold is given by

$$
U_{t}=\Phi\left(\begin{array}{l}
x_{1}(t) \\
x_{2}(t)
\end{array}\right) \cdot f_{n_{0}}+h\left(x_{1}, x_{2}, 0\right)
$$


Let $z=x_{1}-i x_{2}$ and $\Psi(0)=\left(\Psi_{1}(0), \Psi_{2}(0)\right)^{T}$. Notice that $p_{1}=\Phi_{1}+i \Phi_{2}$, it follows from (A5) that

$$
U_{t}=\frac{1}{2}\left(p_{1} z+\overline{p_{1} z}\right) \cdot f_{n_{0}}+W(z, \bar{z})
$$

where $W(z, \bar{z})=h\left(\frac{z+\bar{z}}{2}, \frac{i(z-\bar{z})}{2}, 0\right)$. Denote

$$
W(z, \bar{z})=W_{20} \frac{z^{2}}{2}+W_{11} z \bar{z}+W_{02} \frac{\bar{z}^{2}}{2}+\ldots
$$

Furthermore, by Wu [38], z satisfies

$$
\dot{z}=i z^{*} \omega^{*} z+g(z, \bar{z})
$$

where

$$
\begin{aligned}
g(z, \bar{z}) & =\left(\Psi_{1}(0)-i \Psi_{2}(0)\right)\left\langle F\left(U_{t}, 0\right), f_{n_{0}}\right\rangle \\
& =\left(\Psi_{1}(0)-i \Psi_{2}(0)\right)\left\langle f\left(U_{t}, \omega^{*}\right), f_{n_{0}}\right\rangle,
\end{aligned}
$$

and setting

$$
g(z, \bar{z})=g_{20} \frac{z^{2}}{2}+g_{11} z \bar{z}+g_{02} \frac{\bar{z}^{2}}{2}+g_{21} \frac{z^{2} \bar{z}}{2}+\ldots
$$

From A6 and (A7), we have

$$
\begin{aligned}
u_{1 t}(-1) & =\frac{1}{2}\left(z e^{-i z^{*} \omega^{*}}+\bar{z} e^{i z^{*} \omega^{*}}\right) b_{n_{0}}+W_{20}^{(1)}(-1) \frac{z^{2}}{2}+W_{11}^{(1)}(-1) z \bar{z}+W_{02}^{(1)}(-1) \frac{\bar{z}^{2}}{2}+\cdots \\
u_{2 t}(0) & =\frac{1}{2}\left(\xi_{1} z+\bar{\xi}_{1} \bar{z}\right) b_{n_{0}}+W_{20}^{(2)}(0) \frac{z^{2}}{2}+W_{11}^{(2)}(0) z \bar{z}+W_{02}^{(2)}(0) \frac{\bar{z}^{2}}{2}+\cdots \\
u_{3 t}(0) & =\frac{1}{2}\left(\xi_{2} z+\bar{\xi}_{2} \bar{z}\right) b_{n_{0}}+W_{20}^{(3)}(0) \frac{z^{2}}{2}+W_{11}^{(3)}(0) z \bar{z}+W_{02}^{(3)}(0) \frac{\bar{z}^{2}}{2}+\cdots
\end{aligned}
$$

Hence,

$$
\begin{aligned}
\left\langle f\left(U_{t}, \omega^{*}\right), f_{n_{0}}\right\rangle & =\frac{z^{2}}{2} \omega^{*}\left(\begin{array}{c}
-\frac{1}{2} \mu e^{-i z^{*} \omega^{*}} \xi_{1} \\
\frac{1}{2} \mu e^{-i z^{*} \omega^{*}} \xi_{1} \\
-\frac{1}{2} \beta \xi_{2}^{2}
\end{array}\right) \Gamma+z \bar{z} \omega^{*}\left(\begin{array}{c}
-\frac{1}{4} \mu\left(e^{-i z^{*} \omega^{*}} \bar{\xi}_{1}+e^{i z^{*} \omega^{*}} \xi_{1}\right) \\
\frac{1}{4} \mu\left(e^{-i z^{*} \omega^{*}} \bar{\xi}_{1}+e^{i z^{*} \omega^{*}} \xi_{1}\right) \\
-\frac{1}{2} \beta \xi_{2} \bar{\xi}_{2}
\end{array}\right) \Gamma \\
& +\frac{\bar{z}^{2}}{2} \omega^{*}\left(\begin{array}{c}
-\frac{1}{2} \mu e^{i z^{*} \omega^{*}} \bar{\xi}_{1} \\
\frac{1}{2} \mu e^{i z^{*} \omega^{*} \bar{\xi}_{1}} \\
-\frac{1}{2} \beta \bar{\xi}_{2}^{2}
\end{array}\right) \Gamma+\frac{z^{2} \bar{z}}{2} \omega^{*}\left(\begin{array}{c}
-\mu \kappa_{1} \\
\mu \kappa_{1} \\
-\beta \kappa_{2}
\end{array}\right)
\end{aligned}
$$


with

$$
\begin{aligned}
\Gamma= & \int_{0}^{l \pi} b_{n_{0}}^{3} d x \\
\kappa_{1}= & \frac{1}{2} e^{i z^{*} \omega^{*}} \int_{0}^{l \pi} W_{20}^{(2)}(0) b_{n_{0}}^{2} d x+e^{-i z^{*} \omega^{*}} \int_{0}^{l \pi} W_{11}^{(2)}(0) b_{n_{0}}^{2} d x \\
& +\frac{1}{2} \bar{\xi}_{1} \int_{0}^{l \pi} W_{20}^{(1)}(-1) b_{n_{0}}^{2} d x+\xi_{1} \int_{0}^{l \pi} W_{11}^{(1)}(-1) b_{n_{0}}^{2} d x \\
\kappa_{2}= & \bar{\xi}_{2} \int_{0}^{l \pi} W_{20}^{(3)}(0) b_{n_{0}}^{2} d x+2 \xi_{2} \int_{0}^{l \pi} W_{11}^{(3)}(0) b_{n_{0}}^{2} d x .
\end{aligned}
$$

Notice that $\int_{0}^{l \pi}\left(\cos \frac{n}{l} x\right)^{3} d x=0$ for $\forall n \in \mathbb{N}, \int_{0}^{l \pi}\left(\cos \frac{n}{l} x\right)^{3} d x=1$ for $n=0$. Let $\left(\Psi_{1}, \Psi_{2}, \Psi_{3}\right)=\Psi_{1}(0)-i \Psi_{2}(0)$. comparing the coefficients with A10, we obtain

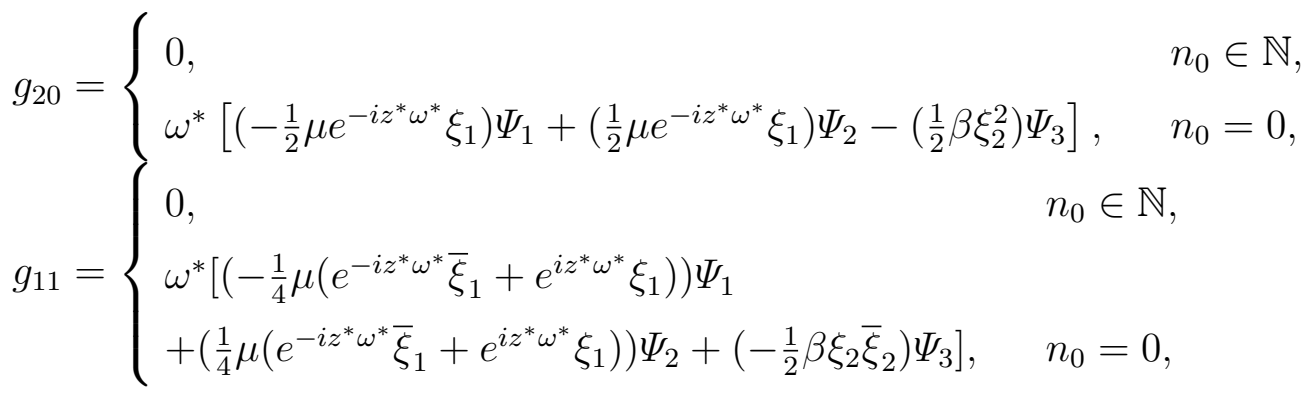

$$
\begin{aligned}
& g_{02}=\bar{g}_{20} \text {, } \\
& g_{21}=\omega^{*}\left(-\mu \kappa_{1} \Psi_{1}+\mu \kappa_{1} \Psi_{2}-\beta \kappa_{2} \Psi_{3}\right), \quad n_{0} \in\{0, \mathbb{N}\} .
\end{aligned}
$$

Since there are $W_{20}(\theta)$ and $W_{11}(\theta)$ in $g_{21}$ for $\theta \in\left[-\tau^{*}, 0\right]$, we still need to compute them. It follows from (A7) that

$$
\begin{gathered}
\dot{W}(z, \bar{z})=W_{20} z \dot{z}+W_{11} \dot{z} \bar{z}+W_{11} z \dot{\bar{z}}+W_{02} \dot{z \bar{z}}+\ldots, \\
A\left(\omega^{*}\right) W=A\left(\omega^{*}\right) W_{20} \frac{z^{2}}{2}+A\left(\omega^{*}\right) W_{11} z \bar{z}+A\left(\omega^{*}\right) W_{02} \frac{\bar{z}^{2}}{2}+\ldots .
\end{gathered}
$$

In addition, By [38],

$$
\dot{W}=A\left(\omega^{*}\right) W+H(z, \bar{z})
$$

and

$$
H(z, \bar{z})=H_{20}(\theta) \frac{z^{2}}{2}+H_{11}(\theta) z \bar{z}+H_{02}(\theta) \frac{\bar{z}^{2}}{2}+\ldots
$$

obviously, Thus, for $-\tau^{*} \leq \theta<0$,

$$
H_{20}(\theta)= \begin{cases}0, & n_{0} \in \mathbb{N} \\ -\frac{1}{2}\left(g_{20} p_{1}(\theta)+\overline{g_{02}} p_{2}(\theta)\right) \cdot f_{0}, & n_{0}=0\end{cases}
$$


and

$$
H_{11}(\theta)= \begin{cases}0, & n_{0} \in \mathbb{N}, \\ -\frac{1}{2}\left(g_{11} p_{1}(\theta)+\overline{g_{11}} p_{2}(\theta)\right) \cdot f_{0}, & n_{0}=0 .\end{cases}
$$

For $\theta=0, H(z, \bar{z})=f\left(U_{t}, \omega^{*}\right)-\Phi\left(\Psi,\left\langle f\left(U_{t}, \omega^{*}\right), f_{n}\right\rangle\right) \cdot f_{n}$, then

$$
\begin{aligned}
& H_{20}(0)= \begin{cases}\widetilde{F}_{z z}^{\prime \prime}, & n_{0} \in \mathbb{N}, \\
\widetilde{F}_{z z}^{\prime \prime}-\frac{1}{2}\left(g_{20} p_{1}(0)+\overline{g_{02}} p_{2}(0)\right) \cdot f_{0}, & n_{0}=0,\end{cases} \\
& H_{11}(0)= \begin{cases}\widetilde{F}_{z \bar{z}}^{\prime \prime}, & n_{0} \in \mathbb{N}, \\
\widetilde{F}_{z \bar{z}}^{\prime \prime}-\frac{1}{2}\left(g_{11} p_{1}(0)+\overline{g_{11}} p_{2}(0)\right) \cdot f_{0}, & n_{0}=0 .\end{cases}
\end{aligned}
$$

Expanding the above series and comparing the coefficients, we obtain

$$
\left(2 i z^{*} \omega^{*} I-A\left(\omega^{*}\right)\right) W_{20}(\theta)=H_{20}(\theta), \quad-A\left(\omega^{*}\right) W_{11}(\theta)=H_{11}(\theta) .
$$

Then A18 have unique solutions $W_{20}$ and $W_{11}$ in $P_{S}(\mathcal{C})$, given by

$$
W_{20}(\theta)=\left(2 i z^{*} \omega^{*} I-A\left(\omega^{*}\right)\right)^{-1} H_{20}(\theta), \quad W_{11}(\theta)=-A\left(\omega^{*}\right)^{-1} H_{11}(\theta) .
$$

Solving for $W_{20}(\theta)$ and $W_{11}(\theta)$, we obtain

$$
\begin{aligned}
& W_{20}(\theta)=\frac{1}{2}\left(\frac{i g_{20}}{z^{*} \omega^{*}} p_{1}(\theta)+\frac{i \bar{g}_{02}}{3 z^{*} \omega^{*}} p_{2}(\theta)\right) \cdot f_{n_{0}}+E_{1} e^{2 i z^{*} \omega^{*} \theta}, \\
& W_{11}(\theta)=\frac{1}{2}\left(-\frac{i g_{11}}{z^{*} \omega^{*}} p_{1}(\theta)+\frac{i \bar{g}_{11}}{z^{*} \omega^{*}} p_{2}(\theta)\right) \cdot f_{n_{0}}+E_{2},
\end{aligned}
$$

Therefore, set $\theta=0$, we can deduce

$$
\begin{aligned}
& 2 i z^{*} \omega^{*} E_{1}-\omega^{*} D \Delta E_{1}-\left.L\left(\omega^{*}\right)\left(E_{1} e^{2 i z^{*} \omega^{*} \theta}\right)\right|_{\theta=0}=\widetilde{F}_{z z}^{\prime \prime}, \\
& -\omega^{*} D \Delta E_{2}-\left.L\left(\omega^{*}\right)\left(E_{2} e^{2 i z^{*} \omega^{*} \theta}\right)\right|_{\theta=0}=\widetilde{F}_{z \bar{z}}^{\prime \prime},
\end{aligned}
$$

where $\widetilde{F}_{z z}^{\prime \prime}=\sum_{n=0}^{\infty}\left\langle\widetilde{F}_{z z}^{\prime \prime}, f_{n}\right\rangle \cdot f_{n}=\sum_{n=0}^{\infty}\left\langle\widetilde{F}_{z z}^{\prime \prime}, f_{n}\right\rangle b_{n}$ and $\widetilde{F}_{z \bar{z}}^{\prime \prime}=\sum_{n=0}^{\infty}\left\langle\widetilde{F}_{z \bar{z}}^{\prime \prime}, f_{n}\right\rangle \cdot f_{n}=\sum_{n=0}^{\infty}\left\langle\widetilde{F}_{z \bar{z}}^{\prime \prime}, f_{n}\right\rangle b_{n}$, $E_{1}=\sum_{n=0}^{\infty} E_{1}^{n} \cdot f_{n}=\sum_{n=0}^{\infty} E_{1}^{n} b_{n}$, and $E_{2}=\sum_{n=0}^{\infty} E_{2}^{n} \cdot f_{n}=\sum_{n=0}^{\infty} E_{2}^{n} b_{n}$, from A20p, we have

$$
\begin{aligned}
& 2 i z^{*} \omega^{*} E_{1}^{n}-\omega^{*} D \Delta E_{1}^{n}-\left.L\left(\omega^{*}\right)\left(E_{1}^{n} e^{2 i z^{*} \omega^{*} \theta}\right)\right|_{\theta=0}=\left\langle\widetilde{F}_{z z}^{\prime \prime}, f_{n}\right\rangle, \\
& -\omega^{*} D \Delta E_{2}^{n}-\left.L\left(\omega^{*}\right)\left(E_{2}^{n} e^{2 i z^{*} \omega^{*} \theta}\right)\right|_{\theta=0}=\left\langle\widetilde{F}_{z \bar{z}}^{\prime \prime}, f_{n}\right\rangle .
\end{aligned}
$$

That is,

$$
\begin{aligned}
& E_{1}^{n}=J_{1}^{n}\left\langle\widetilde{F}_{z z}^{\prime \prime}, f_{n}\right\rangle, \\
& E_{2}^{n}=J_{2}^{n}\left\langle\widetilde{F}_{z \bar{z}}^{\prime \prime}, f_{n}\right\rangle,
\end{aligned}
$$


where

$$
\begin{aligned}
& J_{1}^{n}=\frac{1}{\omega^{*}}\left(\begin{array}{ccc}
2 i z^{*}+d_{1} \frac{n^{2}}{l^{2}}+d+\mu I^{*} e^{-2 i z^{*} \omega^{*}} & \mu S^{*}-\gamma & -\alpha+\alpha e^{-d \tau} e^{-2 i z^{*} \omega^{*} \tau^{*}} \\
-\mu I^{*} e^{-2 i z^{*} \omega^{*}} & 2 i z^{*}+d_{2} \frac{n^{2}}{l^{2}} & 0 \\
0 & 0 & 2 i z^{*}+d_{3} \frac{n^{2}}{l^{2}}+2 \beta y^{*}-\alpha e^{-d \tau} e^{-2 i z^{*} \omega^{*} \tau^{*}}
\end{array}\right)^{-1}, \\
& J_{2}^{n}=\frac{1}{\omega^{*}}\left(\begin{array}{ccc}
d_{1} \frac{n^{2}}{l^{2}}+d+\mu I^{*} & \mu S^{*}-\gamma & -\alpha+\alpha e^{-d \tau} \\
-\mu I^{*} & d_{2} \frac{n^{2}}{l^{2}} & 0 \\
0 & 0 & d_{3} \frac{n^{2}}{l^{2}}+2 \beta y^{*}-\alpha e^{-d \tau}
\end{array}\right)^{-1},
\end{aligned}
$$

and

$$
\begin{gathered}
\left\langle\widetilde{F}_{z z}^{\prime \prime}, f_{n}\right\rangle= \begin{cases}\frac{1}{\sqrt{l \pi}} \widetilde{F}_{20}, & n_{0} \neq 0, n=0, \\
\frac{1}{\sqrt{2 l l}} \widetilde{F}_{20}, & n_{0} \neq 0, n=2 n_{0}, \\
\frac{1}{\sqrt{l \pi}} \widetilde{F}_{20}, & n_{0}=0, n=0, \\
0, & \text { other },\end{cases} \\
\left\langle\widetilde{F}_{z \bar{z}}^{\prime \prime}, f_{n}\right\rangle= \begin{cases}\frac{1}{\sqrt{l \pi}} \widetilde{F}_{11}, & n_{0} \neq 0, n=0, \\
\frac{1}{\sqrt{2 l \pi}} \widetilde{F}_{11}, & n_{0} \neq 0, n=2 n_{0}, \\
\frac{1}{\sqrt{l \pi}} \widetilde{F}_{11}, & n_{0}=0, n=0, \\
0, & \text { other, }\end{cases} \\
\widetilde{F}_{20}=\omega^{*}\left(\begin{array}{l}
-\frac{1}{2} \mu e^{-i z^{*} \omega^{*}} \xi_{1} \\
\frac{1}{2} \mu e^{-i z^{*} \omega^{*}} \xi_{1} \\
-\frac{1}{2} \beta \xi_{2}^{2}
\end{array}\right), \\
\widetilde{F}_{11}=\omega^{*}\left(\begin{array}{l}
-\frac{1}{4} \mu\left(e^{-i z^{*} \omega^{*}} \overline{\xi_{1}}+e^{i z^{*} \omega^{*}} \xi_{1}\right) \\
\frac{1}{4} \mu\left(e^{-i z^{*} \omega^{*}} \overline{\xi_{1}}+e^{i z^{*} \omega^{*}} \xi_{1}\right) \\
-\frac{1}{2} \beta \xi_{2} \bar{\xi}_{2}
\end{array}\right) .
\end{gathered}
$$

Thus, we can determine $W_{20}(\theta)$ and $W_{11}(\theta)$ from A19). Furthermore, we can compute $g_{i j}$ in A11.

\section{REFERENCES}

[1] Kermack, W.O., McKendrik, A.G.: A contribution to the mathematical theory of epidemics. Proc. Roy. Soc. A 115, 700-721 (1927) 
[2] Li, M.Y., Muldowney, J.S.: Global stability for the SEIR model in epidemiology. Math. Biosci. $125,155-164(1995)$

[3] Liu, W.M., Levin, S.A., Iwasa, Y.: Influence of nonlinear incidence rates upon the behavior of SIRS epidemiological models. J. Math. Biol. 23, 187-204 (1986)

[4] Ruan, S., Wang, W.: Dynamical behavior of an epidemic model with a nonlinear incidence rate. J. Differential Equations 188, 135-163 (2003)

[5] Brauer, F.: Models for the spread of universally fatal diseases. J. Math. Biol. 28, 451-462 (1990)

[6] Thieme, H.R.: Epidemic and demographic interaction in the spread of potentially fatal diseases in growing populations. Math. Biosci. 111, 99-130 (1992)

[7] Menalorca, J., Hethcote, H.W.: Dynamic models of infectious diseases as regulators of population sizes. J. Math. Biol. 30, 693-716 (1992)

[8] Hethcote, H.W.: The mathematics of infectious diseases. SIAM Rev. 42, 599-653 (2000)

[9] Anderson, R.M., May, R.M.: Infectious Diseases of Humans Dynamics and Control. Oxford University Press, Oxford (1992)

[10] Hethcote, H.W., Driessche, P.V.D.: Some epidemiological models with nonlinear incidence. J. Math. Biol. 29, 271-287 (1991)

[11] Diekmann, O., Heesterbeek, J.A., Metz, J.A.: On the definition and the computation of the basic reproduction ratio $\mathrm{R} 0$ in models for infectious diseases in inhomogeneous populations. J. Math. Biol. 28, 365-382 (1990)

[12] Kretzschmar, M., Jager, J.C., Reinking, D.P., Van, Z.G., Brouwers, H.: The basic reproduction ratio $\mathrm{R} 0$ for a sexually transmitted disease in a pair formation model with two types of pairs. Math. Biosci. 124, 181-205 (1994)

[13] Cooke, K.L.: Stability analysis for a vector disease model. Rocky Mountain J. Math. 9, 31-42 (1979)

[14] Grossman, Z.: Oscillatory phenomena in a model of infectious diseases. Theoret. Pop. Biol. $18,204-243(1980)$

[15] Saker, S.H.: Stability and Hopf bifurcations of nonlinear delay malaria epidemic model. Nonlinear Anal. Real World Appl. 11, 784-799 (2010)

[16] Khan, Q.J., Krishnan, E.V.: An epidemic model with a time delay in transmission. Appl. Math-Czech. 48, 193-203 (2003) 
[17] Khan, Q.J., Greenhalgh, D.: Hopf bifurcation in epidemic models with a time delay in vaccination. Math. Med. Biol. 16, 113-142 (1999)

[18] Cooke, K.L., Driessche, P.V.D., Zou, X: Interaction of maturation delay and nonlinear birth in population and epidemic models. J. Math. Biol. 39, 332-352 (1999)

[19] Wei, J., Zou, X.: Bifurcation analysis of a population model and the resulting SIS epidemic model with delay. J. Comput. Appl. Math. 197, 169-187 (2006)

[20] Xiao, Y., Chen, L.: An SIS epidemic model with stage structure and a delay. Acta. Math. Appl. Sin-E 18, 607-618 (2002)

[21] Du, Y., Guo, Y., Xiao, P.: Freely-moving delay induces periodic oscillations in a structured SEIR model. Int. J. Bifurcation Chaos 27, 1750122- 1-15 (2017)

[22] Beretta, E., Hara, T., Ma, W., Takeuchi, Y.: Global asymptotic stability of an SIR epidemic model with distributed time delay. Nonlinear Anal. 47, 4107-4115 (2001)

[23] Aiello, W.G., Freedman, H.I.: A time-delay model of single-species growth with stage structure. Math. Biosci. 101, 139-153 (1990)

[24] Cao, Y., Fan, J., Gard, T.C.: The effects of state-dependent time delay on a stage-structured population growth model. Nonlinear Anal. TMA 19, 95-105 (1992)

[25] Huo, H., Li, W., Agarwal, R.P.: Optimal harvesting and stability for two species stagestructured system with cannibalism. Int. J. Appl. Math. 6, 59-79 (2001)

[26] Chattopadhyay, J., Arino, O.: A predator-prey model with disease in the prey. Nonlinear Anal. Real World Appl. 36, 747-766 (1999)

[27] Hethcote, H.W., Wang, W., Ma, Z., Han, L.: A predator-prey model with infected prey. Theoret. Pop. Biol. 66, 259-268 (2004)

[28] Hsieh, Y.H., Hsiao, C.K.: Predator-prey model with disease infection in both populations. Math. Med. Biol. 25, 247-266 (2008)

[29] Shi, X., Cui, J., Zhou, X.: Stability and Hopf bifurcation analysis of an eco-epidemic model with a stage structure. Nonlinear Anal. TMA 74, 1088-1106 (2011)

[30] Monttoni, P. de, Orlandi, E., Tesei, A.: Asymptotic behavior for a system describing epidemics with migration and spatial spread of infection. Nonlinear Anal. 3, 663-675 (1979)

[31] Anita, S., Capasso, V.: A stabilizability problem for a reaction-diffusion system modelling a class of spatially structured epidemic systems. Nonlinear Anal. Real World Appl. 3, 453-464 (2002) 
[32] Peng, R.: Asymptotic profiles of the positive steady state for an SIS epidemic reaction-diffusion model. Part I. J. Differential Equations 247, 1096-1119 (2009)

[33] Busenberg, S.N., Travis, C.C.: Epidemic models with spatial spread due to population migration. J. Math. Biol. 16, 181-198 (1983)

[34] Malchow, H., Petrovskii, S.V., Venturino, E.: Spatiotemporal Patterns in Ecology and Epidemiology: Theory, Models, and Simulation. CRC Press, London (2008)

[35] Capasso, V., Wilson, R.E.: Analysis of a reaction-diffusion system modeling manEenvironment-man epidemics. SIAM J. Appl. Math. 57, 327-346 (1997)

[36] Busenberg, S., Huang, W.: Stability and Hopf Bifurcation for a Population Delay Model with Diffusion Effects. J. Differential Equations 124, 80-107 (1996)

[37] Xu, R., Ma, Z.: An HBV model with diffusion and time delay. J. Theor. Biol. 257, 499-509 (2009)

[38] Wu, J.: Theory and Applications of Partial Functional-Differential Equations. Springer, New York (1996)

[39] Cai, Y., Liu, W., Wang, Y., Wang, W.: Complex dynamics of a diffusive epidemic model with strong Allee effect. Nonlinear Anal. Real World Appl. 14, 1907-1920 (2013)

[40] Song, Y., Peng, Y., Zou, X. Persistence, stability and Hopf bifurcation in a diffusive Ratiodependent predator-prey model with delay. Int. J. Bifurcation Chaos 24, 1450093- 1-18 (2014)

[41] Liu, P.: Periodic solutions in an epidemic model with diffusion and delay. Appl. Math. Comput. 265, 275-291 (2015)

[42] Song, Y., Zou, X.: Spatiotemporal dynamics in a diffusive ratio-dependent predator-prey model near a Hopf-Turing bifurcation point. Comput. Math. Appl. 67, 1978-1997 (2014)

[43] Baurmann, M., Gross, T., Feudel, U.: Instabilities in spatially extended predator-prey systems: spatio-temporal patterns in the neighborhood of Turing-Hopf bifurcations. J. Theor. Biol. 245, 220-229 (2007)

[44] Su, Y., Wei, J., Shi, J.: Bifurcation analysis in a delayed diffusive Nicholsons blowflies equation,. Nonlinear Anal. Real World Appl. 11, 1692-1703 (2010)

[45] Guo, S., Ma, L.: Stability and bifurcation in a delayed reactionCdiffusion equation with Dirichlet boundary condition. J. Nonlinear Sci. 26, 545-580 (2016)

[46] Su, Y., Zou, X.: Transient oscillatory patterns in the diffusive non-local blowfly equation with delay under the zero-flux boundary condition. Nonlinearity 27, 87-104 (2014) 
[47] Faria, T.: Normal forms and Hopf bifurcation for partial differential equations with delays. Trans. Amer. Math. Soc. 352, 2217-2238 (2000)

[48] Hassard, B.D., Kazarinoff, N.D., Wan, Y.H.: Theory and Applications of Hopf Bifurcation. Cambridge University Press, Cambridge (1981)

[49] Yi, F., Wei, J., Shi, J.: Bifurcation and spatiotemporal patterns in a homogeneous diffusive predator-prey system. J. Differential Equations 246, 1944-1977 (2009)

[50] Zhao, J., Wei, J.: Dynamics in a diffusive plankton system with delay and toxic substances effect. Nonlinear Anal. Real World Appl. 22, 66-83 (2015)

[51] Li, X., Ruan, S., Wei, J.: Stability and bifurcation in delay-differential equations with two delays. J. Math. Anal. Appl. 236, 254-280 (1999)

[52] Ruan, S., Wei, J.: On the zeros of transcendental functions with applications to stability of delay differential equations with two delays. Dyn. Contin. Discrete Impuls. Syst. Ser. A Math. Anal. 10, 863-874 (2003)

[53] Chang, X., Wei, J.: Hopf bifurcation and optimal control in a diffusive predator-prey system with time delay and prey harvesting. Nonlinear Anal-Model. 17, 379-409 (2012)

[54] Niu, B., Jiang, W.: Nonresonant Hopf-Hopf bifurcation and a chaotic attractor in neutral functional differential equations. J. Math. Anal. Appl. 398, 362-371 (2014) 